\title{
Molecular Descriptor Analysis of Certain Isomeric Natural Polymers
}

\author{
Maqsood Ahmad, ${ }^{1}$ Muhammad Saeed $\mathbb{D},{ }^{2}$ Muhammad Javaid $\left(\mathbb{D},{ }^{2}\right.$ and Ebenezer Bonyah ${ }^{3}{ }^{3}$ \\ ${ }^{1}$ Department of Mathematics, COMSATS University Islamabad, Lahore Campus, Lahore 54000, Pakistan \\ ${ }^{2}$ Department of Mathematics, School of Science, University of Management and Technology, Lahore 54000, Pakistan \\ ${ }^{3}$ Department of Mathematics Education, Akenten Appiah-Menka University of Skills Training and Entrepreneurial Development, \\ Kumasi 00233, Ghana \\ Correspondence should be addressed to Ebenezer Bonyah; ebbonya@gmail.com
}

Received 1 July 2021; Accepted 7 August 2021; Published 24 August 2021

Academic Editor: Ajaya Kumar Singh

Copyright (C) 2021 Maqsood Ahmad et al. This is an open access article distributed under the Creative Commons Attribution License, which permits unrestricted use, distribution, and reproduction in any medium, provided the original work is properly cited.

\begin{abstract}
Polymers, drugs, and almost all chemical or biochemical compounds are frequently modeled as diverse $\omega$-cyclic, acyclic, bipartite, and polygonal shapes and regular graphs. Molecular descriptors (topological indices) are the numerical quantities and computed from the molecular graph $\Gamma(2 \mathrm{D}$ lattice). These descriptors are highly significant in quantitative structure-property or activity relationship (QSPR and QSAR) modeling that provides the theoretical and the optimal basis to expensive experimental drug design. In this paper, we study three isomeric natural polymers of glucose (polysaccharides), namely, cellulose, glycogen, and amylopectin (starch), having promising pharmaceutical applications, exceptional properties, and fascinating molecular structures. We intend to investigate and compute various closed-form formulas such as $\mathrm{ABC}, \mathrm{GA}$, sum-connectivity $\chi_{(-1 / 2)}, \mathrm{ABC}_{4}, \mathrm{GA}_{5}$, and Sanskruti indices for the aforementioned macromolecules. Also, we present the closed-form formulas for the first, second, modified, and augmented Zagreb indices, inverse and general Randić indices, and symmetric division deg, harmonic, and inverse sum indices. Furthermore, we provide a comparative analysis using 3D graphs for these families of macromolecules to clarify their nature.
\end{abstract}

\section{Introduction}

Cheminformatics is a comparatively new area of information technology that comprises chemistry, mathematics, and other informational sciences that concentrate on gathering, storage, treatment, and examination of chemical data. A molecular descriptor (MD) distinguishes the topology of a molecular graph and is invariant under isomorphism. Some of these descriptors take part in QSAR/QSPR analysis $[1,2]$ which infer about the bioactivities and physicochemical properties of biochemical materials. Various types of distance-based, degree-based, spectral, and polynomial-related descriptors of graphs are well established and extensively studied in the literature. Out of these classes, vertex degree-based descriptors turn out to be the most important and play a phenomenal role in chemical graph theory (CGT). These descriptors are used, in combination, to infer physicochemical, biological, and pharmacological properties such as the stability, chirality, melting point, boiling point, similarity, connectivity, entropy, enthalpy of formation, surface tension, density, critical temperature, and toxicity of chemical compounds in CGT; see $[3,4]$.

Throughout, in this work, $\Gamma$ denotes a simple, finite, and connected graph, whereas $E(\Gamma)$ and $V(\Gamma)$ represent the edge and vertex set of $\Gamma$, respectively. For a vertex $v \in V(\Gamma)$, degree of vertex $v$ is denoted by $d_{v}$, and the sum of the degree of vertices at unit distance from $v$ is represented by $S_{v}$, i.e., $S_{v}=\sum_{w \in N_{\Gamma}(v)} d_{w}$, where $N_{\Gamma}(v)=\{w \in V(\Gamma) \mid v w \in E(\Gamma)\}$. Here, we specify few distinct, significant, and well-studied bond-additive invariants of our concern.

In [5], Gutman and Trinajstić proposed two degreebased invariants known as the first Zagreb index (FZI) $M_{1}$ and the second Zagreb index (SZI) $M_{2}$. These indices initially appeared in the expression of the total $\pi$-electron of the molecular graph and were later applied to study molecular complexity and ZE isomerism. The formulas of $M_{1}, M_{2}$, and the modified Zagreb index (MSZI) are given as 


$$
\begin{aligned}
& M_{1}(\Gamma)=\sum_{u v \in E(\Gamma)}\left(d_{u}+d_{v}\right), \\
& M_{2}(\Gamma)=\sum_{u v \in E(\Gamma)}\left(d_{u} \times d_{v}\right), \\
& { }^{m} M_{2}(\Gamma)=\sum_{u v \in E(\Gamma)} \frac{1}{\left(d_{u} d_{v}\right)} .
\end{aligned}
$$

In [6], Randić offered a very influential invariant which is considered to be a prototype of degree-based invariants and is called Randić index. It is the oldest and extensively studied invariant that measures the amount of branching in the carbon atom skeleton of saturated hydrocarbons $[7,8]$. For a molecular graph $\Gamma$, Randić index is defined as

$$
R(\Gamma)=\sum_{u v \in E(\Gamma)} \frac{1}{\sqrt{d_{u} d_{v}}} .
$$

In [9], Bollobás and Erdös initiated the concept of the general Randić index (GRI), and it is given as

$$
R_{\alpha}(\Gamma)=\sum_{u v \in E(\Gamma)}\left(d_{u} d_{v}\right)^{\alpha}, \quad \alpha \in \mathbb{R} .
$$

Moreover, the inverse Randić index (IRI) is defined by the following formula:

$$
R R_{\alpha}(\Gamma)=\sum_{u v \in E(\Gamma)}\left(d_{u} d_{v}\right)^{\alpha}
$$

In [10], Zhou and Trinajstic instigated the concept of the generalized sum-connectivity index (GSCI) that is defined as follows:

$$
\chi_{\alpha}(\Gamma)=\sum_{u v \in E(\Gamma)}\left(d_{u}+d_{v}\right)^{\alpha}, \quad \alpha \in \mathbb{R}
$$

For $\alpha=(-1 / 2)$ and $\alpha=2$, we have sum-connectivity index $\chi_{(-1 / 2)}$ (SCI) and "hyper-Zagreb index" (HZI) [11], respectively.

In [12], Li and Zheng introduced the first general Zagreb index (FGZI), and it is defined by the following formula:

$$
M_{1}^{\alpha}=\sum_{v \in V(\Gamma)}\left(d_{v}\right)^{\alpha}=\sum_{u v \in E(\Gamma)}\left(d_{u}^{\alpha-1}+d_{v}^{\alpha-1}\right) .
$$

For $\alpha=3$, we obtain the forgotten index (FI). In [13], Azari and Iranmanesh initiated the idea of the generalized Zagreb index (GZI) that is given as

$$
Z_{r, s}(\Gamma)=\sum_{u v \in E(\Gamma)}\left(d_{u}^{r} d_{v}^{s}+d_{u}^{s} d_{v}^{r}\right)
$$

where $r, s \in \mathbb{Z}^{+} \cup\{0,-1\}$.

In [14], Estrada et al. introduced a significant invariant called atom-bond connectivity index $\mathrm{ABC}(\Gamma)$ that proved to be a good predictor for the stability of alkanes and strain energy of cycloalkanes [15, 16]. In [17], Vukičević and Furtula suggested another prominent invariant known as geometric-arithmetic index GA $(\Gamma)$. These indices are defined as follows:

$$
\begin{aligned}
\operatorname{ABC}(\Gamma) & =\sum_{u v \in E(\Gamma)} \sqrt{\frac{d_{u}+d_{v}-2}{d_{u} d_{v}}}, \\
\operatorname{GA}(\Gamma) & =\sum_{u v \in E(\Gamma)} \frac{2 \sqrt{d_{u} d_{v}}}{d_{u}+d_{v}}
\end{aligned}
$$

An interested reader may refer to the surveys [18, 19] regarding Randić and GA indices of graphs, respectively. In $[20,21]$, Ghorbani and Hosseinzadeh and Graovac et al. proposed the fourth version of $\mathrm{ABC}$ and the fifth version of GA that are denoted by $\mathrm{ABC}_{4}$ and $\mathrm{GA}_{5}$, respectively. Likewise, Hosamani introduced the Sanskruti index denoted by SI [22]. These invariants are based on the sum of neighbor's degrees of end vertices and are defined as

$$
\begin{aligned}
\operatorname{ABC}_{4}(\Gamma) & =\sum_{u v \in E(\Gamma)} \sqrt{\frac{S_{u}+S_{v}-2}{S_{u} S_{v}}} \\
\mathrm{GA}_{5}(\Gamma) & =\sum_{u v \in E(\Gamma)} \frac{2 \sqrt{S_{u} S_{v}}}{S_{u}+S_{v}} \\
\mathrm{SI}(\Gamma) & =\sum_{u v \in E(\Gamma)}\left(\frac{S_{u} S_{v}}{S_{u}+S_{v}-2}\right)^{3}
\end{aligned}
$$

For a molecular graph $(\Gamma)$, some other invariants of key importance and related to our concern are SDD (symmetric division deg), HI (harmonic index), ISI (inverse sum index), and AZI (augmented Zagreb index). These indices are defined as follows:

$$
\begin{aligned}
\operatorname{SDD}(\Gamma) & =\sum_{u v \in E(\Gamma)}\left(\frac{d_{u}^{2}+d_{v}^{2}}{d_{u} d_{v}}\right), \\
\mathrm{HI}(\Gamma) & =\sum_{u v \in E(\Gamma)}\left(\frac{2}{d_{u}+d_{v}}\right) \\
\operatorname{ISI}(\Gamma) & =\sum_{u v \in E(\Gamma)}\left(\frac{d_{u} d_{v}}{d_{u}+d_{v}}\right) \\
\operatorname{AZI}(\Gamma) & =\sum_{u v \in E(\Gamma)}\left(\frac{d_{u} d_{v}}{d_{u}+d_{v}-2}\right)^{3}
\end{aligned}
$$

In [23], Ranjini et al. proposed the idea of first, second, and third redefined Zagreb invariants that are given by the following formulas:

$$
\begin{aligned}
& \operatorname{ReZM}_{1}(\Gamma)=\sum_{u v \in E(\Gamma)}\left(\frac{d_{u}+d_{v}}{d_{u} d_{v}}\right), \\
& \operatorname{ReZM}_{2}(\Gamma)=\sum_{u v \in E(\Gamma)}\left(\frac{d_{u} d_{v}}{d_{u}+d_{v}}\right), \\
& \operatorname{ReZM}_{3}(\Gamma)=\sum_{u v \in E(\Gamma)}\left(d_{u} d_{v}\right)\left(d_{u}+d_{v}\right) .
\end{aligned}
$$


It is evident that $\operatorname{ReZM}_{1}=n$ and violates the criteria to be a topological index. Furthermore, $\operatorname{ReZM}_{2}$ is the same as the already defined topological index called the inverse sum index. Consequently, the only novel invariant is $\operatorname{ReZM}_{3}(\Gamma)$ known as redefined Zagreb index (ReZI) and is denoted by $\operatorname{ReZM}(\Gamma)$.

In [24], Deutsch and Klavžar initiated the idea of $M$ polynomial for graph $\Gamma=(V(\Gamma), E(\Gamma))$, and it is mathematically given as

$$
M(\Gamma ; x, y)=f(x, y)=\sum_{i \leq j} m_{i j}(\Gamma) x^{i} y^{j},
$$

where $m_{i j}(\Gamma)$ denotes the number of edges $u v \in E(\Gamma)$, where $\left\{d_{u}, d_{v}\right\}=\{i, j\}$.

Table 1 depicts the relationship between some essential topological indices and the $M$-polynomial, where $D_{x} M=x(\partial M / \partial x), \quad D_{y} M=y(\partial M / \partial y), \quad J(M(x, y))=M$ $(x, x), \quad S_{x} M=\int_{0}^{x}(M(t, y) / t) \mathrm{d} t, \quad S_{y} M=\int_{0}^{y}(M(x, t) / t) \mathrm{d} t$, and $Q_{\alpha} M=x^{\alpha} M$.

Note: all formulas depicted in Table 1 will be calculated at $x=y=1$.

We summarize the relationship of GZI with certain important invariants in Table 2.

Harry Wiener, an American theoretical chemist, observed that invariants estimated from the molecular graph of a chemical compound carry information and properties of that chemical compound. Camarda and Maranas [25] employed the connectivity indices to invent and create the polymers correlated with a certain optimal characteristic. Dendrimers are acknowledged to be the "polymers of the 21st century" due to their increased popularity, which is evident through research articles and patents registered. In [26], Wang et al. provided the closed-form formula for the $k$-connectivity invariant in the class of nanostars and dendrimers. In [27], Ali et al. derived general formulas of certain invariants for some specific polymers such as polyphenylenes, nanostars, and dendrimers. In [28], Shao et al. worked out for the maximum value of the ABC index and provided its characterization in the class of chemically oriented graphs. In [29], Gao et al. figured out the enthalpy and entropy for copper oxide I and copper oxide II. Kang et al. [30], Liu et al. [31], and Gao et al. [32] studied various topological aspects of 2D silicon-carbons, nanotubes, and dendrimers, respectively.

Liu et al. [33] investigated and identified proteins having nucleotide-binding activity using star graph TIs. Ali et al. $[34,35]$ and Du et al. [36] studied and applied some degreebased TIs such as the first Zagreb connection index, ordinary generalized geometric-arithmetic index, general Platt index, and general sum-connectivity index to establish extremal results for alkanes. Hayat et al. [37] performed comparative testing of certain chemical structures (carbon nanotubes, carbon nanocones, and tetrahedral diamond) using various degree-based TIs. Arockiaraj et al. [38] computed variants of Wiener indices for the molecular graphs of coronoid systems, carbon nanocones, and $\mathrm{SiO}_{2}$ nanostructures. In [39], Ahmad et al. computed and compared several invariants of synthetic polymers such as bakelite, vulcanized rubber, and
TABLE 1: Formulas to derive some promising invariants from the $M$-polynomial.

\begin{tabular}{lc}
\hline Topological indices & Formulas derived from the $M$-polynomial \\
\hline FZI $M_{1}$ & $\left(D_{x}+D_{y}\right) M(x, y)$ \\
SZI $M_{2}$ & $\left(D_{x} \cdot D_{y}\right) M(x, y)$ \\
MSZI ${ }^{m} M_{2}$ & $\left(S_{x} \cdot S_{y}\right) M(x, y)$ \\
GRI $R_{\alpha}$ & $D_{x}^{\alpha} \cdot D_{y}^{\alpha} M(x, y)$ \\
IRI $R R_{\alpha}$ & $S_{x}^{\alpha} \cdot S_{y}^{\alpha} M(x, y)$ \\
SDD & $\left(D_{x} S_{y}+S_{x} D_{y}\right) M(x, y)$ \\
HI & $2 S_{x} J M(x, y)$ \\
ISI & $S_{x} J D_{x} D_{y} M(x, y)$ \\
AZI & $S_{x}^{3} Q_{-2} J D_{x}^{3} D_{y}^{3} M(x, y)$ \\
\hline
\end{tabular}

TABLE 2: Few particular cases of GZI.

\begin{tabular}{lc}
\hline Topological index & Corresponding $(r, s)$-Zagreb index \\
\hline FZI $M_{1}(\Gamma)$ & $Z_{1,0}$ \\
SZI $M_{2}(\Gamma)$ & $(1 / 2) Z_{1,1}$ \\
FI $F(\Gamma)$ & $Z_{2,0}$ \\
ReZI ReZM $(\Gamma)$ & $Z_{2,1}$ \\
GFZI $M^{\alpha}(\Gamma)$ & $Z_{\alpha-1,0}$ \\
GRI $R_{\alpha}(\Gamma)$ & $(1 / 2) Z_{\alpha, \alpha}$ \\
SDD index SDD $(\Gamma)$ & $Z_{1,-1}$ \\
\hline
\end{tabular}

acrylic (polymethyl methacrylate) to ascertain a relationship between their physicochemical properties. From their monomers, we develop the polymeric graphs of three closely related natural polymers (isomeric), broadly known as cellulose, glycogen, and amylopectin, to compute certain invariants to anticipate their physicochemical properties. Numerous theoretical, mathematical, and chemical properties of diverse chemical structures based on various invariants obtained from their molecular graphs have been investigated in [40-43].

Polymers pervade every aspect of our daily life, and it is hard to imagine a society without natural as well as synthetic polymers, and they are characterized into four major types based on their molecular chains; see Figure 1.

Typically, almost all food items comprise macromolecules which are some sort of polymers. Most of the food items primarily include naturally occurring polymers (polysaccharides) such as starch and cellulose. The main biological functions of these polysaccharides are nutritional, e.g., energy storage for metabolism (starch and glycogen), and building material (cellulose). Like graphite and diamonds, glycogen, starch, and cellulose are also composed of the same substance but with different structures. We know glycogen, starch, and cellulose are all natural polymers of glucose (carbohydrate) having the same chemical formula $\left(\mathrm{C}_{6} \mathrm{H}_{10} \mathrm{O}_{5}\right)_{n}$. The entire class of natural polymers is made up of smaller segments called monomers (monosaccharides), and glucose is the basic building block for cellulose, glycogen, and starch. They differ from each other based on the glucose type present and the nature of the bond which links the glucose monomers together. Glucose is a type of sugar comprising carbon, hydrogen, and oxygen. These elements bind together to create a hexagonal structure having six carbon atoms (numbered $\mathrm{C}_{1}$ to $\mathrm{C}_{6}$ ) with one of the carbons 

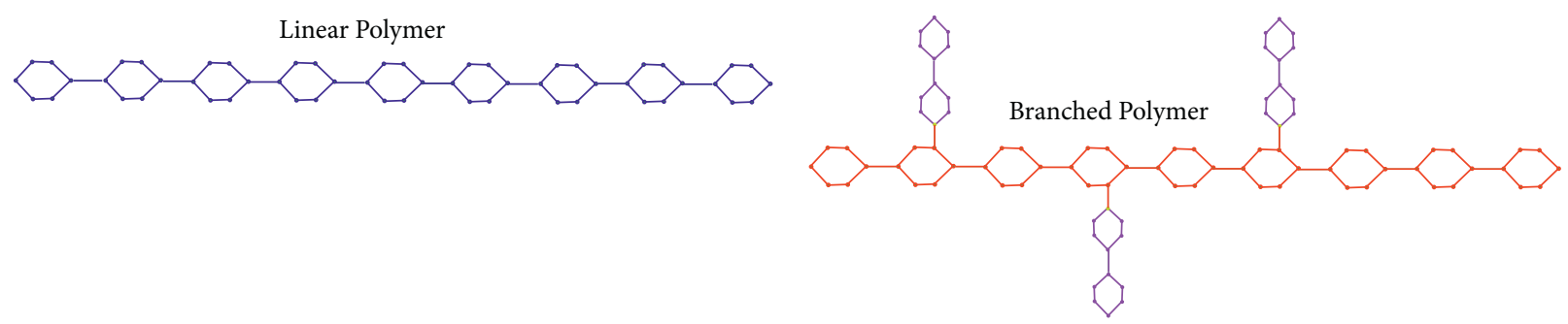

(a)

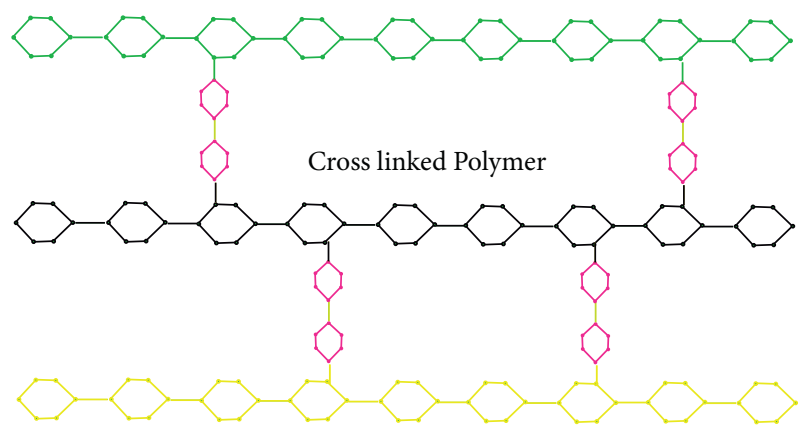

(c)

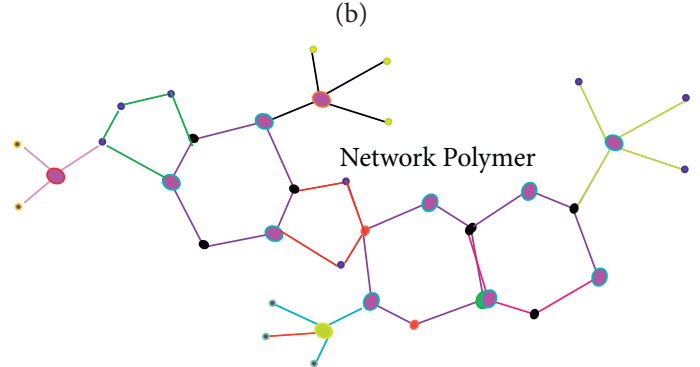

(d)

Figure 1: Polymer characterization with regard to structural chains.

sticking off the end. Distinct glucose rings can be attached at different carbons to produce different types of structures. Some segments of the ring are flipped, causing two different forms of glucose, known as $\alpha$-glucose (alcohol OH attached to $\mathrm{C}_{1}$ is down) and $\beta$-glucose (alcohol $\mathrm{OH}$ attached to $\mathrm{C}_{1}$ is up); see Figure 2. There are two types of bonding, namely, $\alpha\left(\mathrm{C}_{1}-\mathrm{C}_{4}\right)$ and $\alpha\left(\mathrm{C}_{1}-\mathrm{C}_{6}\right)$ glycosidic bonding in amylopectin and glycogen; see Figure 2. Natural polymers, particularly of carbohydrate origin, have been found very promising pharmaceutical applications in different forms [44-46].

\section{Discussion and Construction of the Planar Graph of Cellulose Network $\mathbf{C L}_{m}^{n}$}

Cellulose is among the most abundant, renewable, and biodegradable organic compounds found in nature. Anselme Payen (1838), a French chemist, recognized the existence of cellulose in green plants [47]. It is the main component of tough cell walls that surround plant cells, thus making plant stems, leaves, and branches strong as well as rigid. The rigid structure of cellulose allows plants to stand upright, difficult to digest, and hard to break down. Recently, the government, as well as industry, is highly interested in products from sustainable and renewable energy resources that produce low human health and environmental risks [48]. Cellulose-based materials (cellulosics) are used as key excipients in compounding pharmaceutical objectives and gained immense attraction due to various intriguing features such as low cost, biocompatibility, reproducibility, and recyclability (green technology).

First, we explain the chemical structure of cellulose, in general, and then convert it into a mathematical object called a molecular graph to investigate its properties using tools from graph theory. It comprises over 3, $000 \mathrm{D}$-glucose units that are linked by $\beta\left(\mathrm{C}_{1}-\mathrm{C}_{4}\right)$ glycosidic bonding (see Figure 3$)$ and have general formula $\left(\mathrm{C}_{6} \mathrm{H}_{10} \mathrm{O}_{5}\right)_{n}$. Cellulose is a linear unbranched polymer: unlike glycogen and starch, no coiling occurs. Multiple hydroxyl groups on the glucose ring from one chain create hydrogen-oxygen bonding on the same or a neighboring linear chain (highly cross-linked polymer) that results in the formation of microfibrils having high tensile strength.

Now, we provide the construction, from scratch, for the molecular graph of $\mathrm{CL}_{m}^{n}$. The basic building unit of the cellulose network is $\left(\mathrm{C}_{6} \mathrm{H}_{10} \mathrm{O}_{5}\right)_{2}$, depicted in Figure 3, consisting of three hexagons and one octagon with three pendant edges. Out of these pendants, one is fixed carbon, and the remaining two pendants are $\mathrm{OH}$ (hydroxyl group), one at the upper side and one at the lower side for further bonding. Here, $n$ represents the number of hexagons in basic units, and when we add one monomer to the basic unit, we get 7 hexagons. Similarly, every single addition of the monomer resulted in an increment of four hexagons.

Assume $n$ to be the number of hexagons in one hexagonal chain with $l$ isomeric units and $m$ to be the number of hexagonal chains in cellulose network $\mathrm{CL}_{m}^{n}$. Clearly, the number of hexagons in each chain is odd, and the relation between hexagons in one chain with isomeric units $l$ is given as $n=4 l+3, l=0,1,2,3, \ldots$. Figure 4 elaborates a threedimensional network of cellulose $\mathrm{CL}_{4}^{7}$ along with its planar network. We recognize three types of the polygon in the molecular graph of cellulose, namely, hexagons, octagons, and decagons.

2.1. Results for Cellulose Network $C L_{m}^{n}$. Using simple counting techniques, we observe that the total number of vertices in 


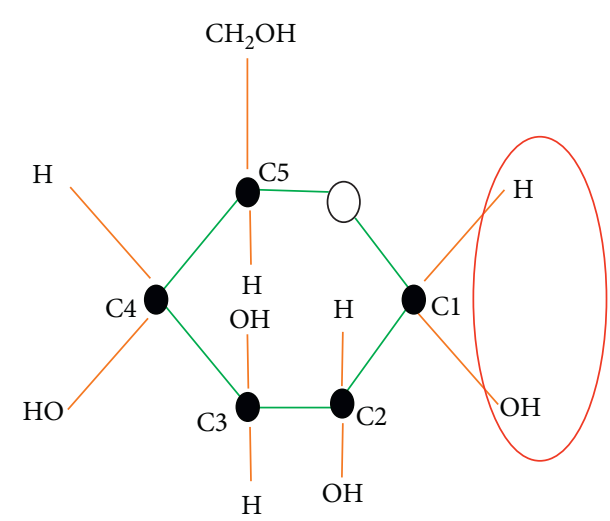

Alpha Glucose

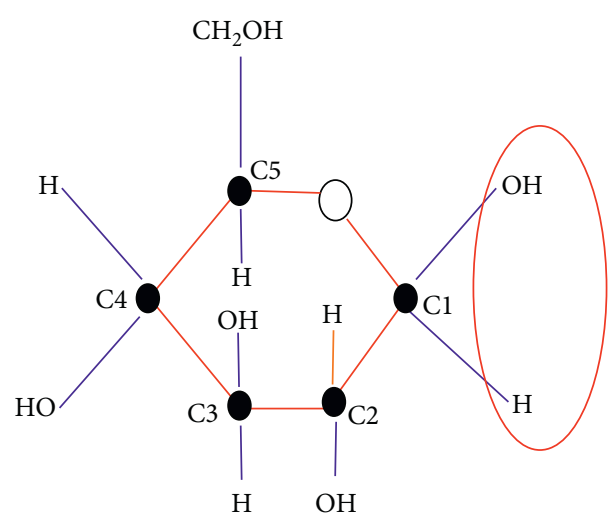

Beta Glucose

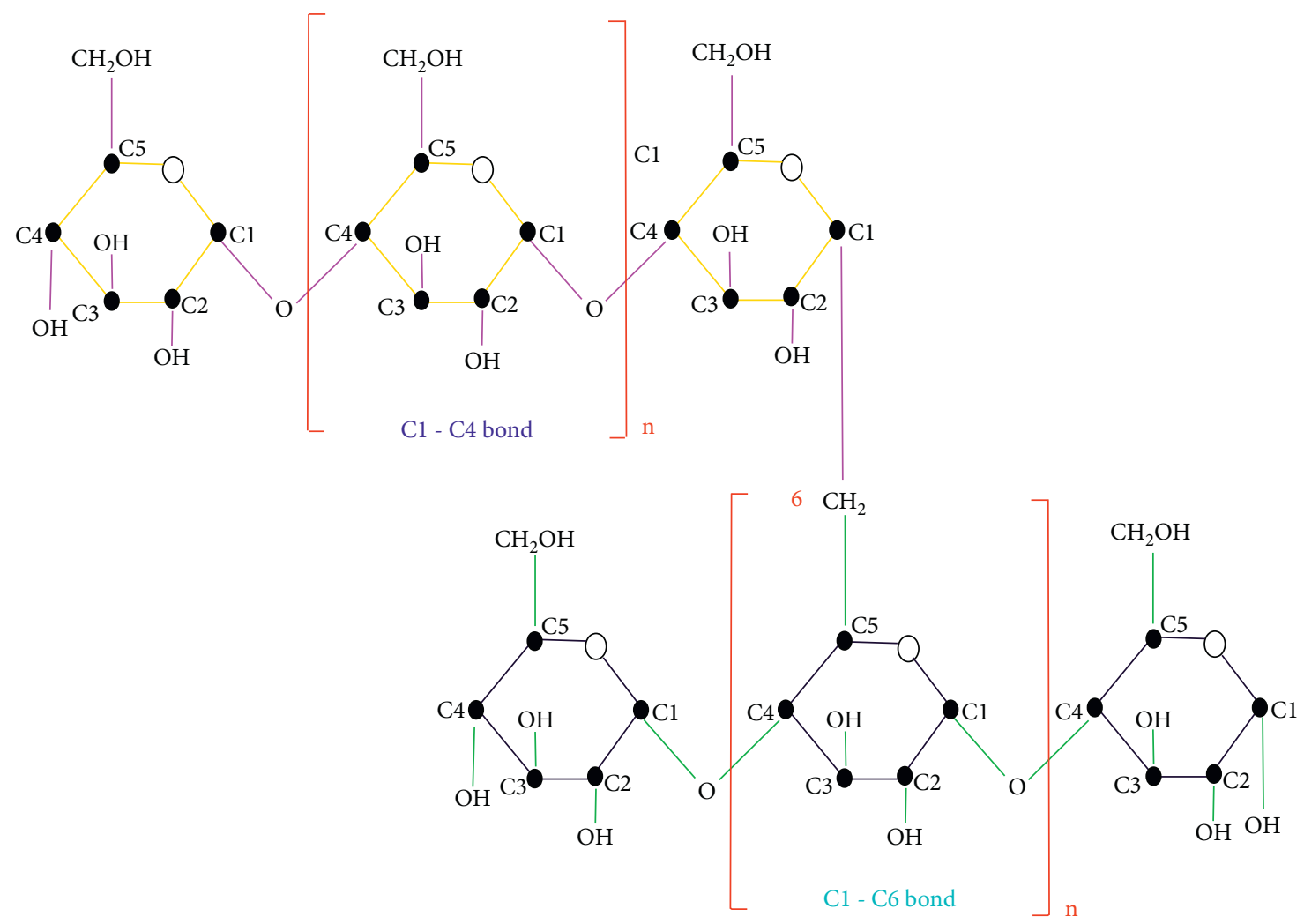

Figure 2: Basic units, $\alpha$ - and $\beta$-glucose, and their linkages.

$\mathrm{CL}_{m}^{n}, n=4 l+3$, is $22 m l+20 m-2$, and the number of edges is $30 m l+25 m-2 l-4$. To compute our results, we require edge partition of edge set $E\left(\mathrm{CL}_{m}^{n}\right)$. There is one and only edge having degrees 1 and 3 of end vertices, i.e., $\left|E_{13}\left(\mathrm{CL}_{m}^{n}\right)\right|=1$. The number of edges with end vertices, each of degree 2 , is $\left|E_{22}\left(\mathrm{CL}_{m}^{n}\right)\right|=4 m+4 l+1$. We detect total edges with degrees 2 and 3 of end vertices as $\left|E_{23}\left(\mathrm{CL}_{m}^{n}\right)\right|=12 m l+12 m-2$. Finally, we identified the number of edges with end vertices, each of degree 3 , as $\left|E_{33}\left(\mathrm{CL}_{m}^{n}\right)\right|=18 m l+9 m-6 l-4$. An edge partition of the cellulose network comprising different parameters is presented in [49]. For the sake of computational ease, we summarized the edge partition of the cellulose network in Table 3.

Theorem 1. Let $C L_{m}^{n}$ be a cellulose network having $m$ hexagonal chains and $n=4 l+3$ hexagons in each chain. The formula for GZI $Z_{r, s}\left(C L_{m}^{n}\right)$ is given as 


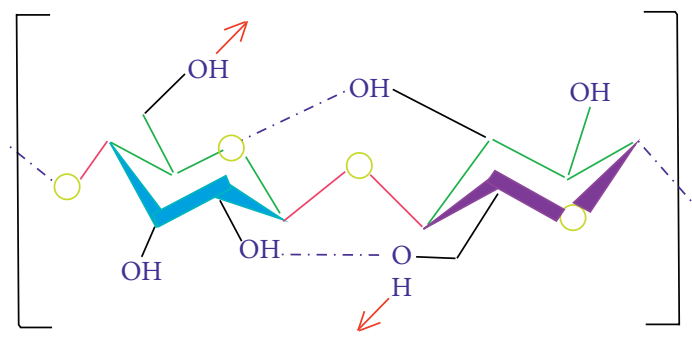

Chemical structure of basic Uinit of Cellulose

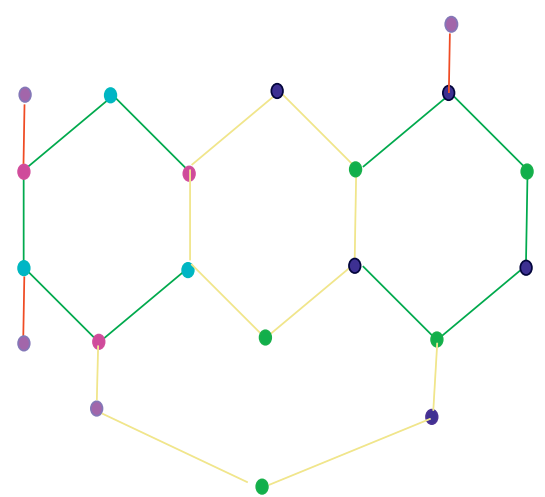

Molecular graph of basic unit of Cellulose

FIgURE 3: 2D skeleton of basic units of cellulose.
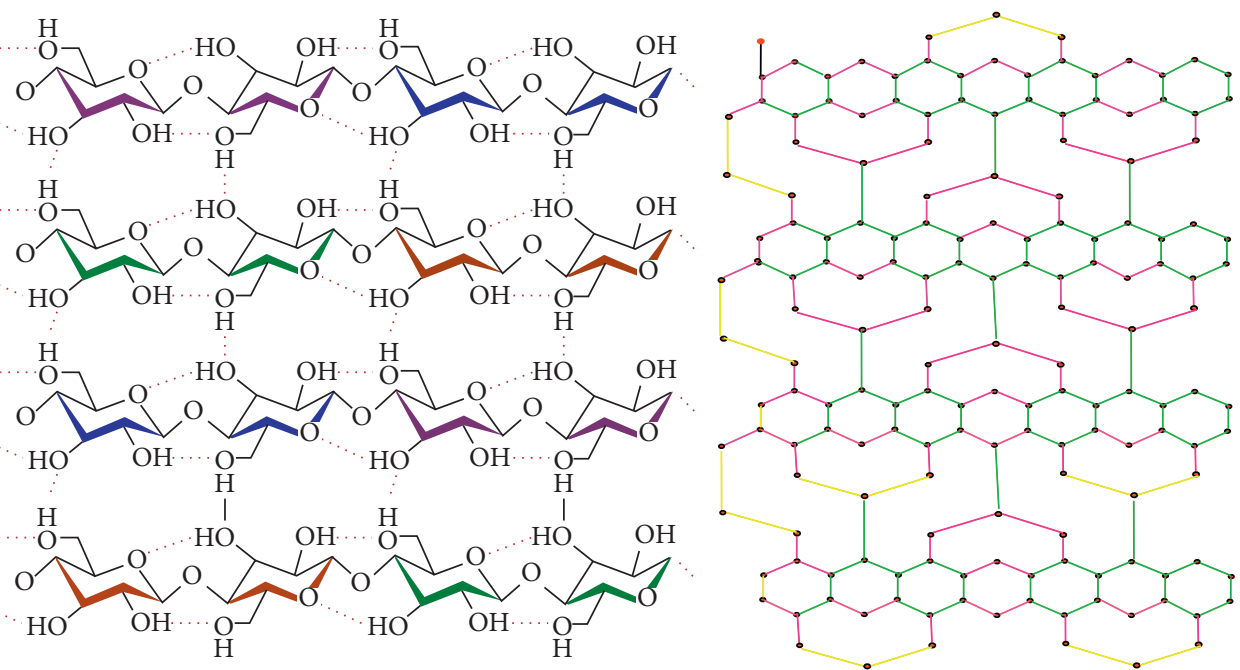

FIgURE 4: Chemical structure and equivalent molecular graph of cellulose $\mathrm{CL}_{4}^{7}$.

TABle 3: Partitioning of the edge set with respect to degrees of end vertices for $\mathrm{CL}_{m}^{n}$.

\begin{tabular}{lcccc}
\hline$\left(d_{v}, d_{w}\right): v w \in E\left(\mathrm{CL}_{m}^{n}\right)$ & $(1,3)$ & $(2,2)$ & $(2,3)$ & $(3,3)$ \\
\hline Number of edges $=\left|E_{(i, j)}\right|$ & 1 & $4 m+4 l+1$ & $12 m l+12 m-2$ & $18 m l+9 m-6 l-4$ \\
\hline
\end{tabular}

$$
\begin{aligned}
Z_{r, s}\left(\mathrm{CL}_{m}^{n}\right)= & \left(4 \times 3^{r+s+2}+2^{r+2} \times 3^{s+1}+2^{s+2} \times 3^{r+1}\right) m l \\
& +\left(2^{r+s+3}+2^{r+2} \times 3^{s+1}+2^{s+2} \times 3^{r+1}+2 \times 3^{r+s+2}\right) m+\left(2^{r+s+3}-4 \times 3^{r+s+1}\right) l \\
& +\left(3^{r}+3^{s}+2^{r+s+1}-2^{r+1} \times 3^{s}-2^{s+1} \times 3^{r}-8 \times 3^{r+s}\right) .
\end{aligned}
$$


Proof. Employing equation (7) and using Table 3, we obtain the desired result as follows:

$$
\begin{aligned}
Z_{r, s}\left(\mathrm{CL}_{m}^{n}\right)= & \sum_{v w \in E\left(\mathrm{CL}_{m}^{n}\right)}\left(d_{v}^{r} d_{w}^{s}+d_{w}^{r} d_{v}^{s}\right)=\sum_{v w \in E_{13}}\left(d_{v}^{r} d_{w}^{s}+d_{w}^{r} d_{v}^{s}\right)+\sum_{v w \in E_{22}}\left(d_{v}^{r} d_{w}^{s}+d_{w}^{r} d_{v}^{s}\right) \\
& +\sum_{v w \in E_{23}}\left(d_{v}^{r} d_{w}^{s}+d_{w}^{r} d_{v}^{s}\right)+\sum_{v w \in E_{33}}\left(d_{v}^{r} d_{w}^{s}+d_{w}^{r} d_{v}^{s}\right) \\
= & \left(3^{s}+3^{r}\right)+(4 m+4 l+1)\left(2^{r} 2^{s}+2^{r} 2^{s}\right)+(12 m l+12 m-2)\left(2^{r} 3^{s}+3^{r} 2^{s}\right) \\
& +(18 m l+9 m-6 l-4)\left(3^{r} 3^{s}+3^{r} 3^{s}\right) \\
= & \left(12\left(2^{r} 3^{s}+2^{s} 3^{r}\right)+36 \times 3^{r+s}\right) m l+\left(12\left(2^{r} 3^{s}+2^{s} 3^{r}\right)+2 \times 3^{r+s+2}+2^{r+s+3}\right) m \\
= & \left(2^{r+s+3}-4 \times 3^{r+s+1}\right) l+\left(3^{r}+3^{s}-2\left(2^{r} 3^{s}+2^{s} 3^{r}\right)+2^{r+s+1}-8 \times 3^{r+s}\right) \\
= & \left(4 \times 3^{r+s+2}+2^{r+2} \times 3^{s+1}+2^{s+2} \times 3^{r+1}\right) m l \\
& +\left(2^{r+s+3}+2^{r+2} \times 3^{s+1}+2^{s+2} \times 3^{r+1}+2 \times 3^{r+s+2}\right) m+\left(2^{r+s+3}-4 \times 3^{r+s+1}\right) l \\
& +\left(3^{r}+3^{s}+2^{r+s+1}-2^{r+1} \times 3^{s}-2^{s+1} \times 3^{r}-8 \times 3^{r+s}\right) .
\end{aligned}
$$

Corollary 1. Using formulas outlined in Table 2 in equation (17), we derived the following results of different TIs:

(1) $M_{1}\left(C L_{m}^{n}\right)=Z_{1,0}\left(C L_{m}^{n}\right)=168 m l+130 m-20 l-26$

(2) $M_{2}\left(C L_{m}^{n}\right)=(1 / 2) Z_{1,1} \quad\left(C L_{m}^{n}\right)=468 m l+338 m-$ $76 l-82$

(3) $F\left(C L_{m}^{n}\right)=Z_{2,0}\left(C L_{m}^{n}\right)=480 m l+350 m-76 l-80$

(4) $\operatorname{ReZM}\left(C L_{m}^{n}\right)=Z_{2,1} \quad\left(C L_{m}^{n}\right)=1332 m l+910 m-$ $260 l-248$

(5) $M^{\alpha}\left(C L_{m}^{n}\right)=Z_{\alpha-1,0} \quad\left(C L_{m}^{n}\right)=\left(16 \times 3^{\alpha}+3 \times 2^{\alpha+1}\right)$ $m l+5\left(2^{\alpha+1}+2 \times 3^{\alpha}\right) m+4\left(2^{\alpha}-3^{\alpha}\right) r+\left(1-3^{\alpha+1}\right)$

(6) $R_{\alpha}\left(C L_{m}^{n}\right)=(1 / 2) \quad Z_{\alpha, \alpha}\left(C L_{m}^{n}\right)=\left(6^{\alpha+1}+9^{\alpha+1}\right) m l+$ $\left(2^{2 \alpha+1}+6^{\alpha+1}+\left(9^{\alpha+1} / 2\right)\right) \quad m+\left(2^{2 \alpha+1}-3^{2 \alpha+2}\right) l-$ $\left(\left(3^{\alpha} / 2\right)+2^{2 \alpha-1}-6^{\alpha}-2 \times 3^{2 \alpha}\right)$

(7) $S D D\left(C L_{m}^{n}\right)=Z_{1,-1}\left(C L_{m}^{n}\right)=62 m l+52 m-4 l-7$
Theorem 2. Let $C L_{m}^{n}$ be a cellulose network having $m$ hexagonal chains and $n=4 l+3$ hexagons in each chain. Then,

(1) $A B C\left(C L_{m}^{n}\right)=(12+6 \sqrt{2}) m l+(6+8 \sqrt{2}) m+$ $(2 \sqrt{2}-4) l+(1 / 6)(2 \sqrt{6}-3 \sqrt{2}-16)$

(2) $G A\left(C L_{m}^{n}\right)=(90+24 \sqrt{6})(\mathrm{ml} / 5)+(65+24 \sqrt{6})(\mathrm{m} /$ 5) $-2 l-(1 / 10)(5 \sqrt{3}-8 \sqrt{6}-30)$

(3) $\operatorname{SCI}\left(C L_{m}^{n}\right)=\chi_{(-1 / 2)}\left(C L_{m}^{n}\right)=((12 / \sqrt{5})+3 \sqrt{6}) m l+$ $(2+(12 / \sqrt{5})+(9 / \sqrt{6})) m+(2-\sqrt{6}) l+(1-(2 /$ $\sqrt{5})-(4 / \sqrt{6}))$

Proof. We determine the required results with the help of Table 3 along with equations (8), (9), and (5), respectively.

$$
\begin{aligned}
1 \mathrm{ABC}\left(\mathrm{CL}_{m}^{n}\right)= & \sum_{u v \in E\left(\mathrm{CL}_{m}^{n}\right)} \sqrt{\frac{d_{u}+d_{v}-2}{d_{u} d_{v}}}=\sum_{u v \in E_{13}\left(\mathrm{CL}_{m}^{n}\right)} \sqrt{\frac{d_{u}+d_{v}-2}{d_{u} d_{v}}} \\
& +\sum_{u v \in E_{22}\left(\mathrm{CL}_{m}^{n}\right)} \sqrt{\frac{d_{u}+d_{v}-2}{d_{u} d_{v}}}+\sum_{u v \in E_{23}\left(\mathrm{CL}_{m}^{n}\right)} \sqrt{\frac{d_{u}+d_{v}-2}{d_{u} d_{v}}} \\
& +\sum_{u v \in E_{33}\left(\mathrm{CL}_{m}^{n}\right)} \sqrt{\frac{d_{u}+d_{v}-2}{d_{u} d_{v}}} \\
= & \sqrt{\frac{2}{3}}+(4 m+4 l+1) \sqrt{\frac{2}{4}}+(12 m l+12 m-2) \sqrt{\frac{3}{6}}+(18 m l+9 m-6 l-4) \sqrt{\frac{4}{9}} \\
= & (12+6 \sqrt{2}) m l+(6+8 \sqrt{2}) m+(2 \sqrt{2}-4) l+\frac{1}{6}(2 \sqrt{6}-3 \sqrt{2}-16),
\end{aligned}
$$




$$
\begin{aligned}
& 2 \mathrm{GA}\left(\mathrm{CL}_{m}^{n}\right)=\sum_{u v \in E\left(\mathrm{CL}_{m}^{n}\right)} \frac{2 \sqrt{d_{u} d_{v}}}{d_{u}+d_{v}}=\sum_{u v \in E_{13}\left(\mathrm{CL}_{m}^{n}\right)} \frac{2 \sqrt{d_{u} d_{v}}}{d_{u}+d_{v}}+\sum_{u v \in E_{22}\left(\mathrm{CL}_{m}^{n}\right)} \frac{2 \sqrt{d_{u} d_{v}}}{d_{u}+d_{v}} \\
& +\sum_{u v \in E_{23}\left(\mathrm{CL}_{m}^{n}\right)} \frac{2 \sqrt{d_{u} d_{v}}}{d_{u}+d_{v}}+\sum_{u v \in E_{33}\left(\mathrm{CL}_{m}^{n}\right)} \frac{2 \sqrt{d_{u} d_{v}}}{d_{u}+d_{v}} \\
& =\left(\frac{2 \sqrt{3}}{4}\right)+(4 m+4 l+1)\left(\frac{2 \sqrt{4}}{4}\right)+(12 m l+12 m-2)\left(\frac{2 \sqrt{6}}{5}\right)+(18 m l+9 m-6 l-4)\left(\frac{2 \sqrt{9}}{6}\right) \\
& =(90+24 \sqrt{6}) \frac{m l}{5}+(65+24 \sqrt{6}) \frac{m}{5}-2 l-\frac{1}{10}(5 \sqrt{3}-8 \sqrt{6}-30), \\
& 3 \chi_{-1 / 2}\left(\mathrm{CL}_{m}^{n}\right)=\sum_{u v \in E\left(\mathrm{CL}_{m}^{n}\right)} \frac{1}{\sqrt{d_{u}+d_{v}}}=\sum_{u v \in E_{13}\left(\mathrm{CL}_{m}^{n}\right)} \frac{1}{\sqrt{d_{u}+d_{v}}}+\sum_{u v \in E_{22}\left(\mathrm{CL}_{m}^{n}\right)} \frac{1}{\sqrt{d_{u}+d_{v}}} \\
& +\sum_{u v \in E_{23}\left(\mathrm{CL}_{m}^{n}\right)} \frac{1}{\sqrt{d_{u}+d_{v}}}+\sum_{u v \in E_{33}\left(\mathrm{CL}_{m}^{n}\right)} \frac{1}{\sqrt{d_{u}+d_{v}}} \\
& =\frac{1}{\sqrt{4}}+\frac{1}{\sqrt{4}}(4 m+4 l+1)+\frac{1}{\sqrt{5}}(12 m l+12 m-2)+\frac{1}{\sqrt{6}}(18 m l+9 m-6 l-4) \\
& =\left(\frac{12}{\sqrt{5}}+3 \sqrt{6}\right) m l+\left(2+\frac{12}{\sqrt{5}}+\frac{9}{\sqrt{6}}\right) m+(2-\sqrt{6}) l+\left(1-\frac{2}{\sqrt{5}}-\frac{4}{\sqrt{6}}\right) .
\end{aligned}
$$

Theorem 3. Let $C L_{m}^{n}$ be a cellulose network having $m$ hexagonal chains and $n=4 l+3$ hexagons in each chain; then, the M-polynomial of $C L_{m}^{n}$ is

$$
M\left(\mathrm{CL}_{m}^{n} ; x, y\right)=x y^{3}+(4 m+4 l+1) x^{2} y^{2}+(12 m l+12 m-2) x^{2} y^{3}+(18 m l+9 m-6 l-4) x^{3} y^{3}
$$

Proof. To calculate the $M$-polynomial of $\mathrm{CL}_{m}^{n}$, we apply equation (15) as follows:

$$
\begin{aligned}
M\left(\mathrm{CL}_{m}^{n} ; x, y\right)= & \sum_{\delta \leq i \leq j \leq \Delta} M_{i j} x^{i} y^{j}=\sum_{1 \leq 3} M_{13} x y^{3} \\
& +\sum_{2 \leq 2} M_{22} x^{2} y^{2}+\sum_{2 \leq 3} M_{23} x^{2} y^{3}+\sum_{3 \leq 3} M_{33} x^{3} y^{3} \\
= & \left|E_{13}\left(\mathrm{CL}_{m}^{n}\right)\right| x y^{3}+\left|E_{22}\left(\mathrm{CL}_{m}^{n}\right)\right| x^{2} y^{2}+\left|E_{23}\left(\mathrm{CL}_{m}^{n}\right)\right| x^{2} y^{3}+\left|E_{33}\left(\mathrm{CL}_{m}^{n}\right)\right| x^{3} y^{3} \\
= & x y^{3}+(4 m+4 l+1) x^{2} y^{2}+(12 m l+12 m-2) x^{2} y^{3} \\
& +(18 m l+9 m-6 l-4) x^{3} y^{3} .
\end{aligned}
$$


Proposition 1. For cellulose network $C L_{m}^{n}$, the formulas for the modified second Zagreb, inverse Randić, harmonic, inverse sum, and augmented Zagreb indices are

(1) ${ }^{m} Z_{2}\left(C L_{m}^{n}\right)=4 m l+4 m+(l / 3)-(7 / 36)$

(2) $R R_{\alpha}\left(C L_{m}^{n}\right)=2\left(6^{1-\alpha}+9^{1-\alpha}\right) m l+\left(4^{1-\alpha}+2 \times 6^{1-\alpha}+\right.$ $\left.9^{1-\alpha}\right) m+\left(4^{1-\alpha}-6 \times 9^{-\alpha}\right) l+\left(3^{-\alpha}+4^{-\alpha}-2 \times 6^{-\alpha}\right.$ $4 \times 9^{-\alpha}$ )
(3) $H I\left(C L_{m}^{n}\right)=(54 / 5) m l+(49 / 5) m-(17 / 15)$

(4) $I S I\left(C L_{m}^{n}\right)=(207 / 5) m l+(319 / 10) m-5 l-(133 / 20)$

(5) $A Z I\left(C L_{m}^{n}\right)=(9633 / 32) m l+(14753 / 64) m-(1163 /$ $32) l-(803 / 16)$

Proof. Consider the $M$-polynomial derived in Theorem 3:

$$
M\left(\mathrm{CL}_{m}^{n} ; x, y\right)=x y^{3}+(4 m+4 l+1) x^{2} y^{2}+(12 m l+12 m-2) x^{2} y^{3}+(18 m l+9 m-6 l-4) x^{3} y^{3}
$$

First, we apply the combination of operators given in derivation Table 1 on the above polynomial as follows:

$$
\begin{aligned}
\left(S_{x} S_{y}\right) M(x, y) & =\frac{1}{3} x y^{3}+\frac{1}{4}(4 m+4 l+1) x^{2} y^{2}+\frac{1}{6}(12 m l+12 m-2) x^{2} y^{3}+\frac{1}{9}(18 m l+9 m-6 l-4) x^{3} y^{3} \\
S_{x}^{\alpha} S_{y}^{\alpha} M(x, y) & =\frac{1}{3^{\alpha}} x y^{3}+\frac{1}{4^{\alpha}}(4 m+4 l+1) x^{2} y^{2}+\frac{1}{6^{\alpha}}(12 m l+12 m-2) x^{2} y^{3}+\frac{1}{9^{\alpha}}(18 m l+9 m-6 l-4) x^{3} y^{3} \\
S_{x} J M(x, y) & =\frac{1}{2}(2 m+2 l+1) x^{4}+\frac{2}{5}(6 m l+6 m-1) x^{5}+\frac{1}{6}(18 m l+9 m-6 l-4) x^{6} \\
S_{x} J D_{x} D_{y} M(x, y) & \left(4 m+4 l+\frac{7}{4}\right) x^{4}+\frac{6}{5}(12 m l+12 m-2) x^{5}+\frac{3}{2}(18 m l+9 m-6 l-4) x^{6}, \\
S_{x}^{3} Q_{-2} J D_{x}^{3} D_{y}^{3} M(x, y) & =\left(32 m+32 l+\frac{91}{8}\right) x^{2}+\frac{216}{27}(12 m l+12 m-2) x^{3}+\frac{729}{64}(18 m l+9 m-6 l-4) x^{4}
\end{aligned}
$$

(1) Modified second Zagreb index $=\left.\left(S_{x} S_{y}\right) M\right|_{x=y=1}=4$ $m l+4 m+(l / 3)-(7 / 36)$

(2) Inverse Randic index $=\left.\left(S_{x}^{\alpha} \cdot S_{y}^{\alpha}\right) M\right|_{x=y=1}=2\left(6^{1-\alpha}+\right.$ $\left.9^{1-\alpha}\right) m l+\left(4^{1-\alpha}+2 \times 6^{1-\alpha}+9^{1-\alpha}\right) m+\left(4^{1-\alpha}-6 \times\right.$ $\left.9^{-\alpha}\right) l+\left(3^{-\alpha}+4^{-\alpha}-2 \times 6^{-\alpha}-4 \times 9^{-\alpha}\right)$

(3) Harmonic index $=\left.2 S_{x} J M(x)\right|_{x=1}=(54 / 5) m l+(49 /$ 5) $m-(17 / 15)$

(4) Inverse sum index $=\left.S_{x} J D_{x} D_{y} M\right|_{x=1}=(207 / 5) m l+$ $(319 / 10) m-5 l-(133 / 20)$

(5) Augmented Zagreb index $=\left.S_{x}^{3} Q_{-2} J D_{x}^{3} D_{y}^{3} M\right|_{x=1}=$ $(9633 / 32) m l+(14753 / 64) m-(1163 / 32) l-(803 / 16)$
To compute $\mathrm{ABC}_{4}\left(\mathrm{CL}_{m}^{n}\right), \mathrm{GA}_{5}\left(\mathrm{CL}_{m}^{n}\right)$, and $\mathrm{SI}\left(\mathrm{CL}_{m}^{n}\right)$, we need the partitioning of edge set $E\left(\mathrm{CL}_{m}^{n}\right)$ with respect to neighbor's degree sum of end vertices for cellulose network $\mathrm{CL}_{m}^{n}$. We observe and identify eight different kinds of edges in $\mathrm{CL}_{m}^{n}$. Now, the partition of the edge set of $\mathrm{CL}_{m}^{n}$ with respect to the degree-sum of the neighbors of the end vertices of each edge is summarized in Table 4.

Theorem 4. Let $C L_{m}^{n}$ be a cellulose network having $m$ hexagonal chains and $n=4 l+3$ hexagons in each chain; then, $A B C_{4}, G A_{5}$, and SI of $C L_{m}^{n}$ are given as follows:

(1)

$$
\begin{aligned}
\mathrm{ABC}_{4}\left(\mathrm{CL}_{m}^{n}\right)= & \frac{1}{126}(12 \sqrt{462}+84 \sqrt{2}+63 \sqrt{14}+84 \sqrt{30}+728) m l \\
& +\frac{1}{1260}(504 \sqrt{35}+855 \sqrt{14}+63 \sqrt{110}+150 \sqrt{462}+90 \sqrt{182}+840 \sqrt{2}+210 \sqrt{30}+4130) m \\
& +\frac{1}{315}(126 \sqrt{35}+450 \sqrt{14}+63 \sqrt{110}-30 \sqrt{462}-210 \sqrt{2}-210 \sqrt{3}-560) l \\
& +\frac{1}{2520}(1185 \sqrt{14}-672 \sqrt{2}-126 \sqrt{110}+420 \sqrt{10}-300 \sqrt{462}-90 \sqrt{182}-420 \sqrt{30}-980) .
\end{aligned}
$$


TABLE 4: Partitioning of the edge set with respect to neighbor's degree sum of end vertices for $\mathrm{CL}_{m}^{n}$.

\begin{tabular}{lc}
\hline$\left(s_{v}, s_{w}\right): v w \in E\left(\mathrm{CL}_{m}^{n}\right)$ & Number of edges \\
\hline$(3,6)$ & 1 \\
$(4,5)$ & $4(m+l)$ \\
$(5,5)$ & 1 \\
$(5,7)$ & $3(m+1)$ \\
$(5,8)$ & $m+4 l-1$ \\
$(6,6)$ & 1 \\
$(6,7)$ & $4 m l+5 m-4 l-5$ \\
$(6,8)$ & $8 m l+3 m+1$ \\
$(7,8)$ & $2 m+1$ \\
$(7,9)$ & $2 m l+2 m-2 l-2$ \\
$(8,8)$ & $4 m l+2 m+8 l-1$ \\
$(8,9)$ & $8 m l+2 m-8 l-2$ \\
$(9,9)$ & $4 m l+m-4 l-1$ \\
Total edges & 1 \\
\hline
\end{tabular}

(2)

$$
\begin{aligned}
\mathrm{GA}_{5}\left(\mathrm{CL}_{m}^{n}\right)= & \frac{1}{6188}(3808 \sqrt{42}+28288 \sqrt{3}+4641 \sqrt{7}+34944 \sqrt{2}+49504) m l \\
& +\frac{1}{278460}(495040 \sqrt{5}+139230 \sqrt{35}+85680 \sqrt{10}+214200 \sqrt{42} \\
& +477360 \sqrt{3}+148512 \sqrt{14}+208845 \sqrt{7}+393120 \sqrt{2}+835380) m \\
& +\frac{1}{7956}(14144 \sqrt{5}+9792 \sqrt{10}-4896 \sqrt{42}-5967 \sqrt{7}-44928 \sqrt{2}+31824) l \\
& +\frac{1}{92820}(53040 \sqrt{3}-69160 \sqrt{2}+46410 \sqrt{35}-28560 \sqrt{10}-71400 \sqrt{42}+24752 \sqrt{14}-69615 \sqrt{7}-92820) .
\end{aligned}
$$

(3)

$$
\begin{aligned}
\mathrm{SI}\left(\mathrm{CL}_{m}^{n}\right)= & \left(\frac{157939036549489}{58436224000}\right) m l+\left(\frac{821225692630075333}{513537536512000}\right) m-\left(\frac{45814497669489}{58436224000}\right) l \\
& -\left(\frac{26341741253143399}{36681252608000}\right) .
\end{aligned}
$$

Proof. Using equation (10) and edge partition presented in

Table 5, we proceed as follows:

$$
\begin{aligned}
1 \mathrm{ABC}_{4}\left(\mathrm{CL}_{m}^{n}\right)= & \sum_{v w \in E\left(\mathrm{CL}_{m}^{n}\right)} \sqrt{\frac{s_{v}+s_{w}-2}{s_{v} s_{w}}}=\left|E_{36}\right| \sqrt{\frac{7}{18}} \\
& +\left|E_{45}\right| \sqrt{\frac{7}{20}}+\left|E_{55}\right| \sqrt{\frac{8}{25}}+\left|E_{57}\right| \sqrt{\frac{10}{35}}+\left|E_{58}\right| \sqrt{\frac{11}{40}} \\
& +\left|E_{66}\right| \sqrt{\frac{10}{36}}+\left|E_{67}\right| \sqrt{\frac{11}{42}}+\left|E_{68}\right| \sqrt{\frac{12}{48}}+\left|E_{78}\right| \sqrt{\frac{13}{56}} \\
& +\left|E_{79}\right| \sqrt{\frac{14}{63}}+\left|E_{88}\right| \sqrt{\frac{14}{64}}+\left|E_{89}\right| \sqrt{\frac{15}{72}}+\left|E_{99}\right| \sqrt{\frac{16}{81}} \\
= & \sqrt{\frac{7}{18}}+\sqrt{\frac{7}{20}}(4 m+4 l)+\frac{2 \sqrt{2}}{5}+\sqrt{\frac{2}{7}}(3 m+3)+\sqrt{\frac{11}{40}}(m+4 l-1) \\
& +\sqrt{\frac{11}{42}}(4 m l+5 m-4 l-5)+\frac{1}{2}(8 m l+3 m+1)+\sqrt{\frac{13}{56}}(2 m-1)
\end{aligned}
$$


TABLE 5: Partitioning of the edge set with respect to neighbor's degree sum of end vertices for $\mathrm{GL}_{m}^{l}$.

\begin{tabular}{lc}
\hline$\left(s_{v}, s_{w}\right): v w \in E\left(\mathrm{GL}_{m}^{l}\right)$ & Number of edges \\
\hline$(3,5)$ & $m+1$ \\
$(3,6)$ & $8 m+m l-l-1$ \\
$(4,5)$ & $2 m+2$ \\
$(5,5)$ & $11 m+m l-l-1$ \\
$(5,6)$ & $12 m+m l-l$ \\
$(5,7)$ & $10 m+m l-l-2$ \\
$(6,6)$ & $28 m+3 m l-3 l-4$ \\
$(6,7)$ & $17 m+2 m l-2 l+1$ \\
$(7,7)$ & $m-1$ \\
Total edges & $9 m(l+10)-9 l-5$ \\
\hline
\end{tabular}

$$
\begin{aligned}
& +\sqrt{\frac{5}{18}}+\sqrt{\frac{14}{63}}(2 m l+2 m-2 l-2)+\sqrt{\frac{7}{32}}(4 m l+2 m+8 l-1) \\
& +\sqrt{\frac{15}{72}}(8 m l+2 m-8 l-2)+\frac{4}{9}(4 m l+m-4 l-2) \\
= & \frac{1}{126}(12 \sqrt{462}+84 \sqrt{2}+63 \sqrt{14}+84 \sqrt{30}+728) m l+\frac{1}{1260}(504 \sqrt{35} \\
& +855 \sqrt{14}+63 \sqrt{110}+150 \sqrt{462}+90 \sqrt{182}+840 \sqrt{2}+210 \sqrt{30}+4130) m \\
& +\frac{1}{315}(126 \sqrt{35}+450 \sqrt{14}+63 \sqrt{110}-30 \sqrt{462}-210 \sqrt{2}-210 \sqrt{3}-560) l \\
& +\frac{1}{2520}(1185 \sqrt{14}-672 \sqrt{2}-126 \sqrt{110}+420 \sqrt{10}-300 \sqrt{462}-90 \sqrt{182}-420 \sqrt{30}-980) .
\end{aligned}
$$

Employing equation (11) and edge partition presented in Table 5, we compute the result in the following manner:

$$
\begin{aligned}
& 2 \mathrm{GA}_{5}\left(\mathrm{CL}_{m}^{n}\right)=\sum_{v w \in E\left(\mathrm{CL}_{m}^{n}\right)} \frac{2 \sqrt{s_{v} s_{w}}}{s_{v}+s_{w}}=\left|E_{36}\right|\left(\frac{2 \sqrt{18}}{9}\right) \\
& +\left|E_{45}\right|\left(\frac{2 \sqrt{20}}{9}\right)+\left|E_{55}\right|\left(\frac{2 \sqrt{25}}{10}\right)+\left|E_{57}\right|\left(\frac{2 \sqrt{35}}{12}\right)+\left|E_{58}\right|\left(\frac{2 \sqrt{40}}{13}\right) \\
& +\left|E_{66}\right|\left(\frac{2 \sqrt{36}}{12}\right)+\left|E_{67}\right|\left(\frac{2 \sqrt{42}}{13}\right)+\left|E_{68}\right|\left(\frac{2 \sqrt{48}}{14}\right)+\left|E_{78}\right|\left(\frac{2 \sqrt{56}}{15}\right) \\
& +\left|E_{79}\right|\left(\frac{2 \sqrt{63}}{16}\right)+\left|E_{88}\right|\left(\frac{2 \sqrt{64}}{16}\right)+\left|E_{89}\right|\left(\frac{2 \sqrt{72}}{17}\right)+\left|E_{99}\right|\left(\frac{2 \sqrt{81}}{18}\right) \\
& =\left(\frac{2 \sqrt{2}}{3}\right)+\left(\frac{4 \sqrt{5}}{9}\right)(4 m+4 l)+1+\left(\frac{\sqrt{35}}{6}\right)(3 m+3)+\left(\frac{4 \sqrt{10}}{13}\right)(m+4 l-1) \\
& +1+\left(\frac{2 \sqrt{42}}{13}\right)(4 m l-5 m-4 l-5)+\left(\frac{4 \sqrt{3}}{7}\right)(8 m l+3 m+1) \\
& +\left(\frac{4 \sqrt{14}}{15}\right)(2 m+1)+\left(\frac{\sqrt{63}}{8}\right)(2 m l+2 m-2 l-2)+(4 m l+2 m+8 l-1) \\
& +\left(\frac{12 \sqrt{2}}{17}\right)(8 m l+2 m-8 l-2)+(4 m l+m-4 l-2)
\end{aligned}
$$




$$
\begin{aligned}
= & \frac{1}{6188}(3808 \sqrt{42}+28288 \sqrt{3}+4641 \sqrt{7}+34944 \sqrt{2}+49504) m l \\
& +\frac{1}{278460}(495040 \sqrt{5}+139230 \sqrt{35}+85680 \sqrt{10}+214200 \sqrt{42}+477360 \sqrt{3} \\
& +148512 \sqrt{14}+208845 \sqrt{7}+393120 \sqrt{2}+835380) m \\
& +\frac{1}{7956}(14144 \sqrt{5}+9792 \sqrt{10}-4896 \sqrt{42}-5967 \sqrt{7}-44928 \sqrt{2}+31824) l \\
& +\frac{1}{92820}(53040 \sqrt{3}-69160 \sqrt{2}+46410 \sqrt{35}-28560 \sqrt{10}-71400 \sqrt{42}+24752 \sqrt{14}-69615 \sqrt{7}-92820)
\end{aligned}
$$

The Sanskruti index SI $\left(\mathrm{CL}_{m}^{n}\right)$ can be calculated as

$$
\begin{aligned}
& 3 \mathrm{SI}\left(\mathrm{CL}_{m}^{n}\right)=\sum_{v w \in E\left(\mathrm{CL}_{m}^{n}\right)}\left(\frac{s_{v} s_{w}}{s_{v}+s_{w}-2}\right)^{3}=\left|E_{36}\right|\left(\frac{18}{7}\right)^{3} \\
& +\left|E_{45}\right|\left(\frac{20}{7}\right)^{3}+\left|E_{55}\right|\left(\frac{25}{8}\right)^{3}+\left|E_{57}\right|\left(\frac{35}{10}\right)^{3}+\left|E_{58}\right|\left(\frac{40}{11}\right)^{3} \\
& +\left|E_{66}\right|\left(\frac{36}{10}\right)^{3}+\left|E_{67}\right|\left(\frac{42}{11}\right)^{3}+\left|E_{68}\right|\left(\frac{48}{12}\right)^{3}+\left|E_{78}\right|\left(\frac{56}{13}\right)^{3} \\
& +\left|E_{79}\right|\left(\frac{63}{14}\right)^{3}+\left|E_{88}\right|\left(\frac{64}{14}\right)^{3}+\left|E_{89}\right|\left(\frac{72}{15}\right)^{3}+\left|E_{99}\right|\left(\frac{81}{16}\right)^{3} \\
& =\left(\frac{18}{7}\right)^{3}+\left(\frac{20}{7}\right)^{3}(4 m+4 l)+\left(\frac{25}{8}\right)^{3}+\left(\frac{7}{2}\right)^{3}(3 m+3)+\left(\frac{40}{11}\right)^{3}(m+4 l-1) \\
& +\left(\frac{18}{5}\right)^{3}+\left(\frac{42}{11}\right)^{3}(4 m l+5 m-4 l-5)+64(8 m l+3 m+1)+\left(\frac{56}{13}\right)^{3}(2 m+1) \\
& +\left(\frac{63}{14}\right)^{3}(2 m l+2 m-2 l-2)+\left(\frac{32}{7}\right)^{3}(4 m l+2 m+8 l-1) \\
& +\left(\frac{24}{5}\right)^{3}(8 m l+2 m-8 l-2)+\left(\frac{81}{16}\right)^{3}(4 m l+m-4 l-2) \\
& =\left(\frac{157939036549489}{58436224000}\right) m l+\left(\frac{821225692630075333}{513537536512000}\right) m \\
& -\left(\frac{45814497669489}{58436224000}\right) l-\left(\frac{26341741253143399}{36681252608000}\right) .
\end{aligned}
$$

\section{Discussion and Construction of the Planar Graph of Glycogen $\mathbf{G L}_{m}^{l}$ and Amylopectin $\mathbf{A M}_{m}^{l}$ Networks}

In the 19th century, Claude Bernard, a prominent French physiologist, discovered glycogen that mainly resides in the liver and muscles. Natural polymers such as cellulose, chitin, proteins, carbohydrates, and glycogen are a great source of energy as they are the key component for life to keep going. Glycogen $\left(\mathrm{C}_{6} \mathrm{H}_{10} \mathrm{O}_{5}\right)_{n}$ is a giant, complex, and highly branched polymer consisting of about 30,000 monomers of glucose. It comprises chains of glucose molecules linearly linked via $\alpha\left(\mathrm{C}_{1}-\mathrm{C}_{4}\right)$ glycosidic linkages, and after every ten to twelve residues, a chain of glucose branches off via $\alpha\left(\mathrm{C}_{1}-\mathrm{C}_{6}\right)$ glycosidic linkages. This latter kind of bonding creates branching and winding patterns in glycogen. On the contrary, cellulose (a close ally) has $\beta\left(\mathrm{C}_{1}-\mathrm{C}_{4}\right)$ glycosidic linkages that produce a 


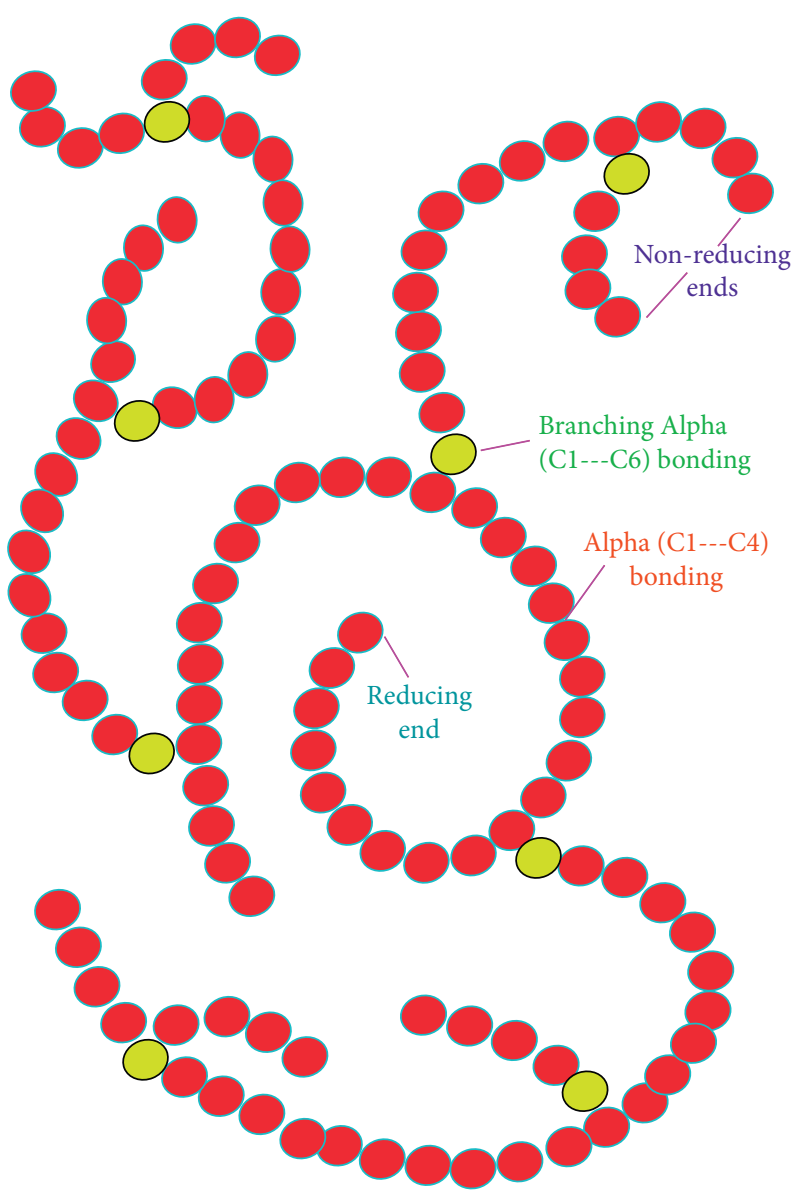

Figure 5: Abstract molecular structure of glycogen.

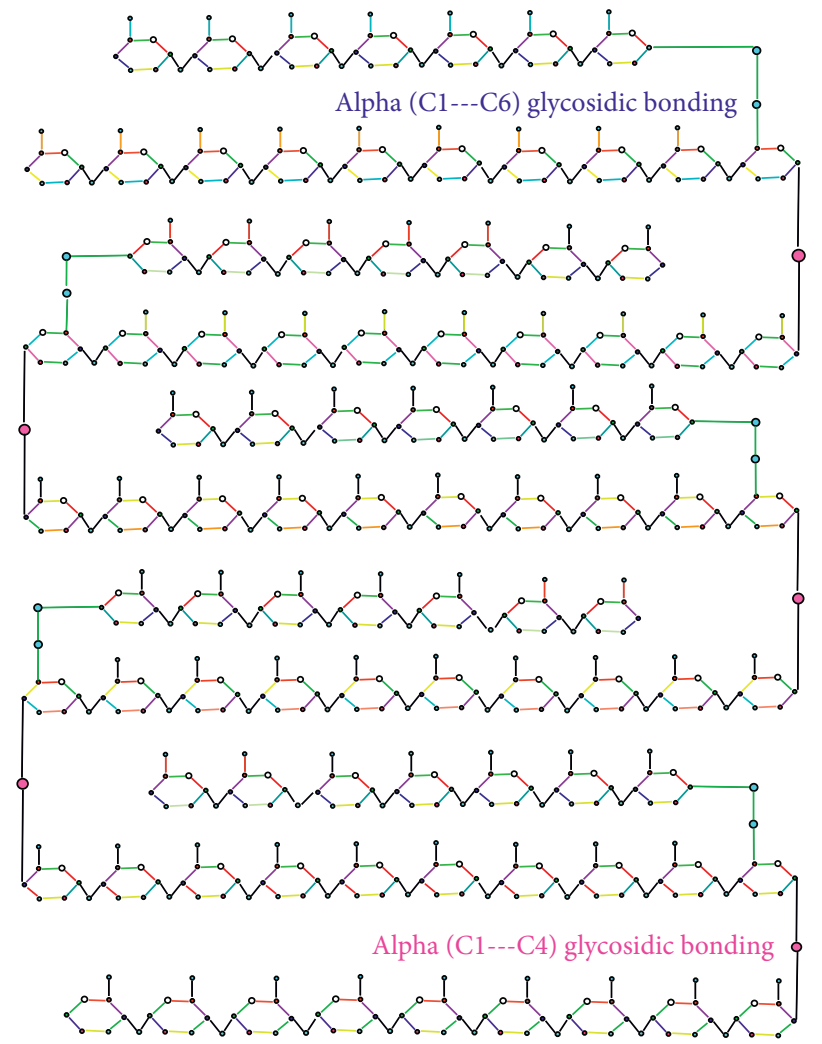

FIGURE 6: Hydrogen-depleted molecular graph of glycogen network $\mathrm{GL}_{6}^{7}$. 
more rigid linear chain and hence cannot be broken down in our stomach. Glycogen has only one reducing end, whereas it has plenty of nonreducing ends; see Figure 5. Glycogen molecules contain glucose as the principal storage reservoir in human and animal cells, and when desired, glycogen is readily processed to release glucose. This self-regulating process maintains the amount of glucose in blood at a constant level even though the supply is uneven. The interaction between glycogen and glucose is at the heart of what is commonly interpreted as the Cori cycle (muscle glycogen $\longrightarrow$ blood lactic acid $\longrightarrow \quad$ liver glycogen $\longrightarrow \quad$ blood glucose $\longrightarrow$ muscle glycogen). Although sufficient investigation has been performed about the regulation of glycogen metabolism by hormones such as insulin, glucagon, and adrenaline [50-52], however, still it is the subject of extensive investigation. In glycogen, approximately after every 10 glucose residues, $\alpha\left(\mathrm{C}_{1}-\mathrm{C}_{6}\right)$ glycosidic bonding occurs which creates the branch. The network of our interest $\mathrm{GL}_{m}^{l}$ is constructed from the glycogen molecule in such a way that it has $m-1$ branches of length $1<l<10$; see Figure 6 .

Amylopectin is an analog of glycogen that has fewer branches and is less compact as compared to glycogen. The helical branching structure gives an open structure to these molecules; as a result, they are easily accessible by enzymes, and so, they can be broken down or assembled quickly. In amylopectin, approximately after every 20 glucose residues, $\alpha\left(\mathrm{C}_{1}-\mathrm{C}_{6}\right)$ glycosidic bonding occurs which creates the branch. The network of our interest $\mathrm{AM}_{m}^{l}$ is constructed from the amylopectin molecule in such a way that it has $m-1$ branches of length $1<l<20$.

3.1. Results for Glycogen Network $G L_{m}^{l}$ and Amylopectin Network $A M_{m}^{l}$. The following lemmas manifest some basic structural properties of the glycogen and amylopectin networks and are essential for upcoming results.

Lemma 1. Let $G L_{m}^{l}$ be the glycogen network shown in Figure 6, with $m-1$ branches each of length $l$; then, the total number of vertices and edges is $8 m(l+10)-8 l-9$ and $9 m(l+10)-9 l-11$, respectively.

Proof. Suppose $V_{\text {deg }}\left(\mathrm{GL}_{m}^{l}\right)$ and $E_{x y}\left(\mathrm{GL}_{m}^{l}\right)$ denote the vertex set and edge set partition and are defined as $V_{d}=\{u \in V$ $\left.\left(\mathrm{GL}_{m}^{l}\right) \mid d_{u}=\operatorname{deg}\right\}$ and $E_{x y}\left(\mathrm{GL}_{m}^{l}\right)=\left\{u v \in E\left(\mathrm{GL}_{m}^{l}\right) \mid\left(d_{u}, d_{v}\right)\right.$ $=(x, y)\}$, respectively. In the molecular graph of glycogen network $\mathrm{GL}_{m}^{l}$, we recognize three kinds of vertices with degrees 1, 2, and 3, i.e., $\delta\left(\mathrm{GL}_{m}^{l}\right)=1$ and $\Delta\left(\mathrm{GL}_{m}^{l}\right)=3$. By applying the basic counting principle, we acquire the partition of the vertex set, and it is presented as $\left|V_{1}\right|=m(9+l)-l$, $\left|V_{2}\right|=42 m+4 m l-4 l-2$, and $\left|V_{3}\right|=29 m+3 m l-3 l-2$. Subsequently, the total number of vertices $\left|V\left(\mathrm{GL}_{m}^{l}\right)\right|$ of the glycogen network is $8 m(l+10)-8 l-4$. Similarly, we identify four types of edges in $\mathrm{GL}_{m}^{l}$ with respect to degrees of end vertices of each edge. Again, employing the basic counting principle, we get the partition of the edge set, and it is given as $\left|E_{13}\right|=9 m+m l-l,\left|E_{22}\right|=12 m+m l-l,\left|E_{23}\right|=$ $60 m+6 m l-6 l-4$, and $\left|E_{33}\right|=9 m+m l-l-1$. Therefore, the total number of edges $\left|E\left(\mathrm{GL}_{m}^{l}\right)\right|$ is $9 m(l+10)-9 l-5$.

Lemma 2 reveals some basic properties of the amylopectin network that are of utmost importance for promising results. Note that we skip the proofs of results for the amylopectin network as they would have been attained by working on the same lines as for the glycogen network.

Lemma 2. Let $A M_{m}^{l}$ be the amylopectin network with $m-1$ branches each of length $l$; then, the total number of vertices and edges is $8 m(l+10)-8 l-4$ and $9 m(l+10)-9 l-5$, respectively.

Without loss of generality and further use, the vertex set and edge set partition of $\mathrm{GL}_{m}^{l}$ and $\mathrm{AM}_{m}^{l}$ are illustrated in Tables 6 and 7, respectively.

Theorem 5. Let $G L_{m}^{l}$ be a glycogen network having $(m-1)$ branches of length $l$, where $1<l<10$. The formula for GZI $Z_{r, s}\left(G L_{m}^{l}\right)$ is given as

$$
\begin{aligned}
Z_{r, s}\left(\mathrm{GL}_{m}^{l}\right)= & \left(3^{s+1}\left(3+5 \times 2^{r+2}\right)+3^{r+1}\left(3+5 \times 2^{s+2}\right)+3 \times 2^{r+s+3}\right) m \\
& +\left(3^{r}\left(1+3^{s}\right)+3^{s}\left(1+53^{r}\right)+2^{r+1} \times 2^{s+1}\right)(m-1) l \\
& +\left(2^{r+2} \times 3^{s}-2^{s+2} \times 3^{r}-2 \times 3^{r+s}\right) .
\end{aligned}
$$

Proof. Employing equation (7) and using Table 6, we obtain

the desired result as follows:

$$
\begin{aligned}
Z_{r, s}\left(\mathrm{GL}_{m}^{l}\right)= & \sum_{v w \in E\left(\mathrm{GL}_{m}^{l}\right)}\left(d_{v}^{r} d_{w}^{s}+d_{w}^{r} d_{v}^{s}\right)=\sum_{v w \in E_{13}}\left(d_{v}^{r} d_{w}^{s}+d_{w}^{r} d_{v}^{s}\right)+\sum_{v w \in E_{22}}\left(d_{v}^{r} d_{w}^{s}+d_{w}^{r} d_{v}^{s}\right) \\
& +\sum_{v w \in E_{23}}\left(d_{v}^{r} d_{w}^{s}+d_{w}^{r} d_{v}^{s}\right)+\sum_{v w \in E_{33}}\left(d_{v}^{r} d_{w}^{s}+d_{w}^{r} d_{v}^{s}\right)
\end{aligned}
$$


TABLE 6: Degree-based partitioning of the vertex set and edge set for $\mathrm{GL}_{m}^{l}$.

\begin{tabular}{lccc}
\hline$d_{v}: v \in V\left(\mathrm{GL}_{m}^{l}\right)$ & Number of vertices & $\left(d_{v}, d_{w}\right): v w \in E\left(\mathrm{GL}_{m}^{l}\right)$ & Number of edges \\
\hline 1 & $m(9+l)-l$ & $(1,3)$ & $9 m+m l-l$ \\
2 & $42 m+4 m l-4 l-2$ & $(2,2)$ & $12 m+m l-l$ \\
3 & $29 m+3 m l-3 l-2$ & $(2,3)$ & $60 m+6 m l-6 l-4$ \\
& & $(3,3)$ & $9 m+m l-l-1$ \\
Total vertices & $8 m(l+10)-8 l-4$ & Total edges & $9 m(l+10)-9 l-5$ \\
\hline
\end{tabular}

TABLe 7: Partitioning of the edge set with respect to the degree of end vertices for $\mathrm{AM}_{m}^{l}$.

\begin{tabular}{|c|c|c|c|}
\hline$d_{v}: v \in V\left(\mathrm{AM}_{m}^{l}\right)$ & Number of vertices & $\left(d_{v}, d_{w}\right): v w \in E\left(\mathrm{AM}_{m}^{l}\right)$ & Number of edges \\
\hline 1 & $19 m+m l-l$ & $(1,3)$ & $19 m+m l-l$ \\
\hline 2 & $82 m+4 m l-4 l-2$ & $(2,2)$ & $22 m+m l-l$ \\
\hline 3 & $59 m+3 m l-3 l-2$ & $\begin{array}{l}(2,3) \\
(3,3)\end{array}$ & $\begin{array}{c}120 m+6 m l-6 l-4 \\
19 m+m l-l-1\end{array}$ \\
\hline Total vertices & $160 m+8 m l-8 l-4$ & Total edges & $180 m+9 m l-9 l-5$ \\
\hline
\end{tabular}

$$
\begin{aligned}
= & (9 m+m l-l)\left(3^{s}+3^{r}\right)+(12 m+m l-l)\left(2^{r} 2^{s}+2^{r} 2^{s}\right) \\
& +(60 m+6 m l-6 l-4)\left(2^{r} 3^{s}+3^{r} 2^{s}\right)+(9 m+m l-l-1)\left(3^{r} 3^{s}+3^{r} 3^{s}\right) \\
= & \left(3^{r+2}+3^{s+2}+3 \times 2^{r+s+3}+60\left(2^{r} 3^{s}+3^{r} 2^{s}\right)+2 \times 3^{r+s+2}\right) m \\
& +\left(3^{r}+3^{s}+2^{r+s+1}+6\left(2^{r} 3^{s}+3^{r} 2^{s}\right)+2 \times 3^{r+s}\right)(m l-l) \\
& +\left(2^{r+2} \times 3^{s}-2^{s+2} \times 3^{r}-3^{r+s+1}\right) \\
= & \left(3^{s+1}\left(3+5 \times 2^{r+2}\right)+3^{r+1}\left(3+5 \times 2^{s+2}\right)+3 \times 2^{r+s+3}\right) m \\
& +\left(3^{r}\left(1+3^{s}\right)+3^{s}\left(1+5 \times 3^{r}\right)+2^{r+1} \times 2^{s+1}\right)(m-1) l \\
& +\left(2^{r+2} \times 3^{s}-2^{s+2} \times 3^{r}-2 \times 3^{r+s}\right) .
\end{aligned}
$$

Corollary 2. From equation (30) of the GZI, we derived the following results of different TIs presented in Table 2:

(1) $M_{1}\left(G L_{m}^{l}\right)=Z_{1,0}\left(G L_{m}^{l}\right)=438 m+44 l(m-1)-26$

(2) $M_{2}\left(G L_{m}^{l}\right)=(1 / 2) Z_{1,1}\left(G L_{m}^{l}\right)=516 m+52 l(m-1)-33$

(3) $F\left(G L_{m}^{l}\right)=Z_{2,0}\left(G L_{m}^{l}\right)=1128 m+114 l(m-1)-70$

(4) $\operatorname{ReZM}\left(G L_{m}^{l}\right)=Z_{2,1}\left(G L_{m}^{l}\right)=2586 m+262 l(m-1)-$ 174

(5) $M^{\alpha}\left(G L_{m}^{l}\right)=Z_{\alpha-1,0}\left(G L_{m}^{l}\right)=\left(42 \times 2^{\alpha}+29 \times 3^{\alpha}+9\right) m$ $+\left(2^{\alpha+2}+3^{\alpha+1}+1\right)(m-1) l-\left(2^{\alpha+1}+2 \times 3^{\alpha+1}\right)$
(6) $R_{\alpha}\left(G L_{m}^{l}\right)=(1 / 2) Z_{\alpha, \alpha}\left(G L_{m}^{l}\right)=\left(3 \times 2^{2 \alpha+2}+3^{\alpha+2}(1+\right.$ $\left.\left.3^{\alpha}\right)+10 \times 6^{\alpha+1}\right) m+\left(2^{2 \alpha}+3^{\alpha}\left(1+3^{\alpha}\right)+6^{\alpha+1}\right)(m-1)$ $l-\left(2^{\alpha+3} \times 3^{\alpha}+2 \times 3^{2 \alpha}\right)$

(7) $\operatorname{SDD}\left(G L_{m}^{l}\right)=Z_{1,-1}\left(G L_{m}^{l}\right)=202 m+(61 / 3)(m-1) l-$ $(32 / 3)$

Theorem 6. Let $A M_{m}^{l}$ be an amylopectin network having $(m-1)$ branches of length $l$, where $1<l<10$. The formula for GZI $Z_{r, s}\left(A M_{m}^{l}\right)$ is given as

$$
\begin{aligned}
Z_{r, s}\left(\mathrm{AM}_{m}^{l}\right)= & \left(19\left(3^{r}+3^{s}\right)+11 \times 2^{r+s+2}+5\left(2^{r+3} 3^{s+1}+3^{r+1} 2^{s+3}\right)+2 \times 3^{r+s+2}\right) m \\
& +\left(3^{r}\left(1+3 \times 2^{s+1}\right)+3^{s}\left(1+3 \times 2^{r+1}\right)\right. \\
& \left.+2\left(2^{r+s}+3^{r+s}\right)\right)(m-1) l-2 \times 3^{r}\left(2^{s+1}+3^{s}\left(2^{r+1}+1\right)\right) .
\end{aligned}
$$


Corollary 3. From equation (31) of the generalized Zagreb index, we derived the following results of different TIs:

(1) $M_{1}\left(A M_{m}^{l}\right)=Z_{1,0}\left(A M_{m}^{l}\right)=878 m+44 l(m-1)-26$

(2) $M_{2}\left(A M_{m}^{l}\right)=(1 / 2) Z_{1,1}\left(A M_{m}^{l}\right)=1036 m+52 l(m-1)$ $-33$

(3) $F\left(A M_{m}^{l}\right)=Z_{2,0}\left(A M_{m}^{l}\right)=2268 m+114 l(m-1)-70$

(4) $\operatorname{ReZM}\left(A M_{m}^{l}\right)=Z_{2,1}\left(A M_{m}^{l}\right)=5206 m+262 l(m-1)$ $-174$

(5) $M^{\alpha}\left(A M_{m}^{l}\right)=Z_{\alpha-1,0}\left(A M_{m}^{l}\right)=\left(41 \times 2^{\alpha+1}+177 \times 3^{\alpha-1}\right.$ $+19) m+\left(2^{\alpha+2}+3^{\alpha+1}+1\right)(m-1) l-\left(2^{\alpha+1}+2 \times 3^{\alpha}\right)$

(6) $R_{\alpha}\left(A M_{m}^{l}\right)=(1 / 2) Z_{\alpha, \alpha}\left(A M_{m}^{l}\right)=\left(38 \times 3^{\alpha}\left(1+3^{\alpha}\right)+\right.$ $\left.2^{\alpha+2}\left(11 \times 2^{\alpha}+20 \times 3^{\alpha+1}\right)\right) m+\left(2^{2 \alpha+1}+2 \times 3^{\alpha}\left(1+3^{\alpha}\right.\right.$ $\left.\left.+3 \times 2^{\alpha+1}\right)\right)(m-1) l-2 \times 3^{\alpha}\left(2^{\alpha+2}+3^{\alpha}\right)$

(7) $\operatorname{SDD}\left(A M_{m}^{l}\right)=Z_{1,-1}\left(A M_{m}^{l}\right)=(1216 / 3) m+(61 / 3)(m$ $-1) l-(32 / 3)$
Theorem 7. Let $G L_{m}^{l}$ be a glycogen network having $(m-1)$ branches of length $l$, where $1<l<10$; then,

(1) $A B C\left(G L_{m}^{l}\right)=3(12 \sqrt{2}+\sqrt{6}+2) m+(1 / 6)(21 \sqrt{2}+$ $2 \sqrt{6}+4)(m-1) l-((6 \sqrt{2}+2) / 3)$

(2) $G A\left(G L_{m}^{l}\right)=((9 \sqrt{3} / 2)+24 \sqrt{6}+21) m+((12 \sqrt{6} / 5)+$ $(\sqrt{3} / 2)+2)(m-1) l-((8 \sqrt{6}-5) / 5)$

(3) $\operatorname{SCI}\left(G L_{m}^{l}\right)=\chi_{(-1 / 2)}\left(G L_{m}^{l}\right)=((21 / 2)+3 \sqrt{(3 / 2)}+12$ $\sqrt{5}) m+((6 / \sqrt{5})+(1 / \sqrt{6})+1)(m-1) l+((5 \sqrt{6}-$ $24 \sqrt{5}) / 30)$

Proof. Using Table 6 and equations (8), (9), and (5), respectively, we compute the desired result as given in the following:

$$
\begin{aligned}
\text { (1) } \mathrm{ABC}\left(\mathrm{GL}_{m}^{l}\right)= & \sum_{u v \in E\left(\mathrm{GL}_{m}^{l}\right)} \sqrt{\frac{d_{u}+d_{v}-2}{d_{u} d_{v}}}=\sum_{u v \in E_{13}\left(\mathrm{GL}_{m}^{l}\right)} \sqrt{\frac{d_{u}+d_{v}-2}{d_{u} d_{v}}} \\
& +\sum_{u v \in E_{22}\left(\mathrm{GL}_{m}^{l}\right)} \sqrt{\frac{d_{u}+d_{v}-2}{d_{u} d_{v}}}+\sum_{u v \in E_{23}\left(\mathrm{GL}_{m}^{l}\right)} \sqrt{\frac{d_{u}+d_{v}-2}{d_{u} d_{v}}} \\
& +\sum_{u v \in E_{33}\left(\mathrm{GL}_{m}^{l}\right)} \sqrt{\frac{d_{u}+d_{v}-2}{d_{u} d_{v}}} \\
= & (9 m+m l-l) \sqrt{\frac{2}{3}+(12 m+m l-l) \sqrt{\frac{2}{4}}+(60 m+6 m l-6 l-4) \sqrt{\frac{3}{6}}} \\
& +(9 m+m l-l-1) \sqrt{\frac{4}{9}} \\
= & 3(12 \sqrt{2}+\sqrt{6}+2) m+\frac{1}{6}(21 \sqrt{2}+2 \sqrt{6}+4)(m-1) l-\left(\frac{6 \sqrt{2}+2}{3}\right),
\end{aligned}
$$$$
\text { (2) } \mathrm{GA}\left(\mathrm{GL}_{m}^{l}\right)=\sum_{u v \in E\left(\mathrm{GL}_{m}^{l}\right)} \frac{2 \sqrt{d_{u} d_{v}}}{d_{u}+d_{v}}=\sum_{u v \in E_{13}\left(\mathrm{GL}_{m}^{l}\right)} \frac{2 \sqrt{d_{u} d_{v}}}{d_{u}+d_{v}}+\sum_{u v \in E_{22}\left(\mathrm{GL}_{m}^{l}\right)} \frac{2 \sqrt{d_{u} d_{v}}}{d_{u}+d_{v}}
$$$$
+\sum_{u v \in E_{23}\left(\mathrm{GL}_{m}^{l}\right)} \frac{2 \sqrt{d_{u} d_{v}}}{d_{u}+d_{v}}+\sum_{u v \in E_{33}\left(\mathrm{GL}_{m}^{l}\right)} \frac{2 \sqrt{d_{u} d_{v}}}{d_{u}+d_{v}}
$$$$
=\left(\frac{2 \sqrt{3}}{4}\right)(9 m+m l-l)+(12 m+m l-l)\left(\frac{2 \sqrt{4}}{4}\right)+(60 m+6 m l-6 l-4)\left(\frac{2 \sqrt{6}}{5}\right)
$$$$
+(9 m+m l-l-1)\left(\frac{2 \sqrt{9}}{6}\right)
$$$$
=\left(\frac{9 \sqrt{3}}{2}+24 \sqrt{6}+21\right) m+\left(\frac{12 \sqrt{6}}{5}+\frac{\sqrt{3}}{2}+2\right)(m-1) l-\left(\frac{8 \sqrt{6}-5}{5}\right),
$$ 


$$
\text { (3) } \begin{aligned}
\chi_{(-1 / 2)}\left(\mathrm{GL}_{m}^{l}\right)= & \sum_{u v \in E\left(\mathrm{GL}_{m}^{l}\right)} \frac{1}{\sqrt{d_{u}+d_{v}}}=\sum_{u v \in E_{13}\left(\mathrm{GL}_{m}^{l}\right)} \frac{1}{\sqrt{d_{u}+d_{v}}}+\sum_{u v \in E_{22}\left(\mathrm{GL}_{m}^{l}\right)} \frac{1}{\sqrt{d_{u}+d_{v}}} \\
& +\sum_{u v \in E_{23}\left(\mathrm{GL}_{m}^{l}\right)} \frac{1}{\sqrt{d_{u}+d_{v}}}+\sum_{u v \in E_{33}\left(\mathrm{GL}_{m}^{l}\right)} \frac{1}{\sqrt{d_{u}+d_{v}}} \\
= & \frac{1}{\sqrt{4}}(9 m+m l-l)+\frac{1}{\sqrt{4}}(12 m+m l-l)+\frac{1}{\sqrt{5}}(60 m+6 m l-6 l-4) \\
& +\frac{1}{\sqrt{6}}(9 m+m l-l-1) \\
= & \left(\frac{21}{2}+3 \sqrt{\frac{3}{2}}+12 \sqrt{5}\right) m+\left(\frac{6}{\sqrt{5}}+\frac{1}{\sqrt{6}}+1\right)(m-1) l+\left(\frac{5 \sqrt{6}-24 \sqrt{5}}{30}\right) .
\end{aligned}
$$

Theorem 8. Let $A M_{m}^{l}$ be an amylopectin network having $(m-1)$ branches of length $l$, where $1<l<10$; then,

(1) $A B C\left(A M_{m}^{l}\right)=(38+213 \sqrt{2}+19 \sqrt{6})(m / 3)+(4+$ $21 \sqrt{2}+2 \sqrt{6})((m-1) l / 6)+(3 \sqrt{2}+1)(2 / 3)$

(2) $G A\left(A M_{m}^{l}\right)=(19 \sqrt{3}+96 \sqrt{6}+82)(m / 2)+(5 \sqrt{3}+$ $24 \sqrt{6}+20)((m-1) l / 10)-((8 \sqrt{6}+5) / 5)$

(3) $\operatorname{SCI}\left(A M_{m}^{l}\right)=\chi_{-1 / 2}\left(A M_{m}^{l}\right)=(144 \sqrt{5}+19 \sqrt{6}+123)$ $(m / 6)+(30 \sqrt{5}+5 \sqrt{6}+30) \quad((m-1) l / 30)+((24$ $\sqrt{5}+5 \sqrt{6}) / 30)$
In the next theorem, we compute the $M$-polynomial of glycogen network $\mathrm{GL}_{m}^{l}$, which will eventually be used to formulate closed-form formulas of certain TIs of our interest.

Theorem 9. Let $G L_{m}^{l}$ be the glycogen network consisting of $(m-1)$ branches where each branch has length $l$; then, $M$ polynomial of $G L_{m}^{l}$ is

$$
M\left(\mathrm{GL}_{m}^{l} ; x, y\right)=(9 m+m l-l) x y^{3}+(12 m+m l-l) x^{2} y^{2}+(60 m+6 m l-6 l-4) x^{2} y^{3}+(9 m+m l-l-1) x^{3} y^{3}
$$

Proof. To calculate the $M$-polynomial of $\mathrm{GL}_{m}^{l}$, we apply equation (15):

$$
\begin{aligned}
M\left(\mathrm{GL}_{m}^{l} ; x, y\right) & =\sum_{\delta \leq i \leq j \leq \Delta} M_{i j} x^{i} y^{j}=\sum_{1 \leq 3} M_{13} x y^{3}+\sum_{2 \leq 2} M_{22} x^{2} y^{2}+\sum_{2 \leq 3} M_{23} x^{2} y^{3}+\sum_{3 \leq 3} M_{33} x^{3} y^{3} \\
& =\left|E_{13}\left(\mathrm{GL}_{m}^{l}\right)\right| x y^{3}+\left|E_{22}\left(\mathrm{GL}_{m}^{l}\right)\right| x^{2} y^{2}+\left|E_{23}\left(\mathrm{GL}_{m}^{l}\right)\right| x^{2} y^{3}+\left|E_{33}\left(\mathrm{GL}_{m}^{l}\right)\right| x^{3} y^{3} \\
& =(9 m+m l-l) x y^{3}+(12 m+m l-l) x^{2} y^{2}+(60 m+6 m l-6 l-4) x^{2} y^{3}+(9 m+m l-l-1) x^{3} y^{3}
\end{aligned}
$$

Proposition 2. For glycogen network $G L_{m}^{l}$, formulas for the modified second Zagreb index, inverse Randic index, symmetric division degree index, harmonic index, inverse sum index, and augmented Zagreb index are as follows:

(1) ${ }^{m} Z_{2}\left(G L_{m}^{l}\right)=17 m+(61 / 36)(m-1) l-(7 / 9)$

(2) $R R_{\alpha}\left(G L_{m}^{l}\right)=\left(3^{2-\alpha}+3 \times 4^{1-\alpha}+10 \times 6^{1-\alpha}+9^{1-\alpha}\right) m+$ $\left(6^{1-\alpha}+9^{1-\alpha}+\left(1 / 3^{\alpha}\right)+\left(1 / 4^{\alpha}\right)\right)(m-1) l-\left(\left(4 / 6^{\alpha}\right)+(1\right.$ $\left.\left./ 9^{\alpha}\right)\right)$
(3) $H I\left(G L_{m}^{l}\right)=(75 / 2) m+(56 / 15)(m-1) l-(29 / 15)$

(4) $\operatorname{ISI}\left(G L_{m}^{l}\right)=(417 / 4) m+(209 / 20)(m-1) l-(63 /$ 10)

(5) $A Z I\left(G L_{m}^{l}\right)=(45369 / 64) m+(4529 / 64)(m-1) l-$ (2777/64)

Proof. Consider the M-polynomial derived in Theorem 9:

$$
M\left(\mathrm{GL}_{m}^{l} ; x, y\right)=(9 m+m l-l) x y^{3}+(12 m+m l-l) x^{2} y^{2}+(60 m+6 m l-6 l-4) x^{2} y^{3}+(9 m+m l-l-1) x^{3} y^{3}
$$


We apply the combination of operators given in derivation Table 1 on the above polynomial as follows:

$$
\begin{aligned}
\left(S_{x} S_{y}\right) M(x, y)= & \frac{1}{3}(9 m+m l-l) x y^{3}+\frac{1}{4}(12 m+m l-l) x^{2} y^{2} \\
& +\frac{1}{6}(60 m+6 m l-6 l-4) x^{2} y^{3}+\frac{1}{9}(9 m+m l-l-1) x^{3} y^{3}, \\
S_{x}^{\alpha} S_{y}^{\alpha} M(x, y)= & \frac{1}{3^{\alpha}}(9 m+m l-l) x y^{3}+\frac{1}{4^{\alpha}}(12 m+m l-l) x^{2} y^{2} \\
& +\frac{1}{6^{\alpha}}(60 m+6 m l-6 l-4) x^{2} y^{3}+\frac{1}{9^{\alpha}}(9 m+m l-l-1) x^{3} y^{3}, \\
2 S_{x} J M(x, y)= & \frac{1}{2}(21 m+2 m l-2 l) x^{4}+\frac{2}{5}(60 m+6 m l-6 l-4) x^{5}+\frac{1}{3}(9 m+m l-l-1) x^{6}, \\
S_{x} J D_{x} D_{y} M(x, y)= & \frac{1}{4}(75 m+7 m l-7 l) x^{4}+\frac{6}{5}(60 m+6 m l-6 l-4) x^{5}+\frac{3}{2}(9 m+m l-l-1) x^{6}, \\
S_{x}^{3} Q_{-2} J D_{x}^{3} D_{y}^{3} M(x, y)= & \frac{27}{8}(9 m+m l-l) x^{2}+8(12 m+m l-l) x^{2} \\
& +8(60 m+6 m l-6 l-4) x^{3}+\frac{729}{64}(9 m+m l-l-1) x^{4} .
\end{aligned}
$$

(1) Modified second Zagreb index $=\left.\left(S_{x} S_{y}\right) M\right|_{x=y=1}=$ $17 m-(61 / 36)(m-1) l-(7 / 9)$

(2) Inverse Randić index $=\left.\left(S_{x}^{\alpha} \cdot S_{y}^{\alpha}\right) M\right|_{x=y=1}=\left(3^{2-\alpha}+\right.$ $\left.3 \times 4^{1-\alpha}+10 \times 6^{1-\alpha}+9^{1-\alpha}\right) m+\left(6^{1-\alpha}+9^{1-\alpha}+(1 /\right.$ $\left.\left.3^{\alpha}\right)+\left(1 / 4^{\alpha}\right)\right)(m-1) l-\left(\left(4 / 6^{\alpha}\right)+\left(1 / 9^{\alpha}\right)\right)$

(3) Harmonic index $=\left.2 S_{x} J M(x)\right|_{x=1}=(75 / 2) m+(56 /$ 15) $(m-1) l-(29 / 15)$
(4) Inverse sum index $=\left.S_{x} J D_{x} D_{y} M\right|_{x=1}=(417 / 4) m+$ $(209 / 20)(m-1) l-(63 / 10)$

(5) Augmented Zagreb index $=\left.S_{x}^{3} Q_{-2} J D_{x}^{3} D_{y}^{3} M\right|_{x=1}=$ $(45369 / 64) m+(4529 / 64)(m-1) l-(2777 / 64)$

Theorem 10. Let $A M_{m}^{l}$ be the amylopectin network consisting of $(m-1)$ branches where each branch has length $l$; then, M-polynomial of $A M_{m}^{l}$ is

$$
M\left(\mathrm{AM}_{m}^{l} ; x, y\right)=(19 m+m l-l) x y^{3}+(22 m+m l-l) x^{2} y^{2}+(120 m+6 m l-6 l-4) x^{2} y^{3}+(19 m+m l-l-1) x^{3} y^{3}
$$

Proposition 3. For amylopectin network $A M_{m}^{l}$, formulas for the modified second Zagreb index, inverse Randic index, symmetric division degree index, harmonic index, inverse sum index, and augmented Zagreb index are as follows:

(1) ${ }^{m} Z_{2}\left(A M_{m}^{l}\right)=(611 / 18) m-(61 / 36)(m-1) l-(7 / 9)$

(2) $R R_{\alpha}\left(A M_{m}^{l}\right)=\left(19 \times 3^{-\alpha}\left(1+3^{-\alpha}\right)+11 \times 2^{1-\alpha}+20 \times\right.$ $\left.6^{1-\alpha}\right) m+\left(3^{-\alpha}\left(1+3^{-\alpha}\right)+2^{-2 \alpha}+6^{1-\alpha}\right)(m-1) l-(4$ $\left.\times 6^{-\alpha}+3^{-2 \alpha}\right)$

(3) $H I\left(A M_{m}^{l}\right)=(449 / 6) m+(56 / 15)(m-1) l-(29 / 15)$

(4) $\operatorname{ISI}\left(A M_{m}^{l}\right)=(835 / 4) m+(209 / 20)(m-1) l-(63 / 10)$
(5) $A Z I\left(A M_{m}^{l}\right)=(90659 / 64) m+(4529 / 64)(m-1) l-$ $(2777 / 64)$

Again, to calculate $\mathrm{ABC}_{4}\left(\mathrm{CL}_{m}^{n}\right), \mathrm{GA}_{5}\left(\mathrm{CL}_{m}^{n}\right)$, and $\mathrm{SI}\left(\mathrm{CL}_{m}^{n}\right)$, we require the partitioning of edge set $E\left(\mathrm{GL}_{m}^{l}\right)$ with respect to neighbor's degree sum of end vertices for glycogen network $\mathrm{GL}_{m}^{l}$. We observe and identify eight different types of edges in $\mathrm{GL}_{m}^{l}$. Now, the partition of the edge set of $\mathrm{GL}_{m}^{l}$ with respect to the degree-sum of the neighbors of the end vertices of each edge is summarized in Table 5. 
TABLE 8: Partitioning of the edge set with respect to neighbor's degree sum of end vertices for $\mathrm{AM}_{m}^{l}$.

\begin{tabular}{lc}
\hline$\left(s_{v}, s_{w}\right): v w \in E\left(\mathrm{AM}_{m}^{l}\right)$ & Number of edges \\
\hline$(3,5)$ & $m+1$ \\
$(3,6)$ & $18 m+m l-l-1$ \\
$(4,5)$ & $2 m+2$ \\
$(5,5)$ & $21 m+m l-l-1$ \\
$(5,6)$ & $22 m+m l-l$ \\
$(5,7)$ & $20 m+m l-l-2$ \\
$(6,6)$ & $58 m+3 m l-3 l-4$ \\
$(6,7)$ & $37 m+2 m l-2 l+1$ \\
$(7,7)$ & $m-1$ \\
Total edges & $180 m+9 m l-9 l-5$ \\
\hline
\end{tabular}

Similarly, the neighbor's degree sum edge partition for amylopectin is depicted in Table 8 .
Theorem 11. Let $G L_{m}^{l}$ be the glycogen network consisting of $(m-1)$ branches where each branch has length $l$; then, $A B C_{4}$, $G A_{5}$, and SI of $G L_{m}^{l}$ are given as follows:

(1)

$$
\begin{aligned}
\mathrm{ABC}_{4}\left(\mathrm{GL}_{m}^{l}\right)= & \frac{1}{210}(1022 \sqrt{10}+580 \sqrt{14}+42 \sqrt{35}+924 \sqrt{2}+252 \sqrt{30}+85 \sqrt{462}+60 \sqrt{3}) m \\
& +\frac{1}{210}(65 \sqrt{14}+84 \sqrt{2}+105 \sqrt{10}+10 \sqrt{462}+21 \sqrt{30})(m-1) l \\
& +\frac{1}{210}(42 \sqrt{35}-98 \sqrt{10}-95 \sqrt{14}-168 \sqrt{2}+5 \sqrt{462}-60 \sqrt{3}) .
\end{aligned}
$$

(2)

$$
\begin{aligned}
\mathrm{GA}_{5}\left(\mathrm{GL}_{m}^{l}\right)= & \frac{1}{5148}(1287 \sqrt{15}+27456 \sqrt{2}+4576 \sqrt{5}+936 \sqrt{30}+8580 \sqrt{35}+13464 \sqrt{42}+205920) m \\
& +\frac{1}{858}(572 \sqrt{2}-156 \sqrt{30}+143 \sqrt{35}+264 \sqrt{42}+3432)(m-1) l \\
& +\frac{1}{468}(117 \sqrt{15}-312 \sqrt{2}+416 \sqrt{5}-156 \sqrt{35}+72 \sqrt{42}-2808)
\end{aligned}
$$

(3)

$$
\mathrm{SI}\left(\mathrm{GL}_{m}^{l}\right)=\left(\frac{2940909850294457}{788889024000}\right) m+\left(\frac{298773851517107}{788889024000}\right)(m-1) l-\left(\frac{216711945260101}{788889024000}\right) .
$$

Proof. Using equation (10) and edge partition presented in Table 5, we proceed as follows:

$$
\text { (1) } \begin{aligned}
\mathrm{ABC}_{4}\left(\mathrm{GL}_{m}^{l}\right)= & \sum_{v w \in E\left(\mathrm{GL}_{m}^{l}\right)} \sqrt{\frac{s_{v}+s_{w}-2}{s_{v} s_{w}}}=\left|E_{35}\right| \sqrt{\frac{6}{15}} \\
& +\left|E_{36}\right| \sqrt{\frac{7}{18}}+\left|E_{45}\right| \sqrt{\frac{7}{20}}+\left|E_{55}\right| \sqrt{\frac{8}{25}}+\left|E_{56}\right| \sqrt{\frac{9}{30}}
\end{aligned}
$$




$$
\begin{aligned}
& +\left|E_{57}\right| \sqrt{\frac{10}{35}}+\left|E_{66}\right| \sqrt{\frac{10}{36}}+\left|E_{67}\right| \sqrt{\frac{11}{42}}+\left|E_{77}\right| \sqrt{\frac{12}{49}} \\
= & \sqrt{\frac{2}{5}}(m+1)+\sqrt{\frac{7}{18}}(8 m+m l-l-1)+\sqrt{\frac{7}{20}}(2 m+2) \\
& +\sqrt{\frac{8}{5}}(11 m+m l-l-2)+\sqrt{\frac{3}{10}}(12 m+m l-l)+\sqrt{\frac{2}{7}}(10 m+m l-l-2) \\
& +\frac{5}{18}(28 m+3 m l-3 l-4)+\sqrt{\frac{11}{42}}(17 m+2 m l-2 l+1)+\frac{\sqrt{12}}{7}(m-1) \\
= & \frac{1}{210}(1022 \sqrt{10}+580 \sqrt{14}+42 \sqrt{35}+924 \sqrt{2}+252 \sqrt{30}+85 \sqrt{462}+60 \sqrt{3}) m \\
& +\frac{1}{210}(65 \sqrt{14}+84 \sqrt{2}+105 \sqrt{10}+10 \sqrt{462}+21 \sqrt{30})(m-1) l \\
& +\frac{1}{210}(42 \sqrt{35}-98 \sqrt{10}-95 \sqrt{14}-168 \sqrt{2}+5 \sqrt{462}-60 \sqrt{3}) .
\end{aligned}
$$

Employing equation (11) and edge partition presented in Table 5, we compute the result in the following manner:

$$
\begin{aligned}
\text { (2) } \mathrm{GA}_{5}\left(\mathrm{GL}_{m}^{l}\right)= & \sum_{v w \in E\left(\mathrm{GL}_{m}^{l}\right)} \frac{2 \sqrt{s_{v} s_{w}}}{s_{v}+s_{w}}=\left|E_{35}\right|\left(\frac{2 \sqrt{15}}{8}\right) \\
& +\left|E_{36}\right|\left(\frac{2 \sqrt{18}}{9}\right)+\left|E_{45}\right|\left(\frac{2 \sqrt{20}}{9}\right)+\left|E_{55}\right|\left(\frac{2 \sqrt{25}}{10}\right)+\left|E_{56}\right|\left(\frac{2 \sqrt{30}}{11}\right) \\
& +\left|E_{57}\right|\left(\frac{2 \sqrt{35}}{12}\right)+\left|E_{66}\right|\left(\frac{2 \sqrt{36}}{12}\right)+\left|E_{67}\right|\left(\frac{2 \sqrt{42}}{13}\right)+\left|E_{77}\right|\left(\frac{2 \sqrt{49}}{14}\right) \\
= & \left.\frac{\sqrt{15}}{4}\right)(m+1)+\left(\frac{2 \sqrt{2}}{3}\right)(8 m+m l-l-1)+\left(\frac{4 \sqrt{5}}{9}\right)(2 m+2) \\
& +(11 m+m l-l-1)+\left(\frac{2 \sqrt{30}}{11}\right)(12 m+m l-l)+\left(\frac{\sqrt{35}}{6}\right)(10 m+m l-l-2) \\
& +(28 m+3 m l-3 l-4)+\left(\frac{2 \sqrt{42}}{13}\right)(17 m+2 m l-2 l+1)+(m-1) \\
& +\frac{1}{468}(117 \sqrt{15}-312 \sqrt{2}+416 \sqrt{5}-156 \sqrt{35}+72 \sqrt{42}-2808) \\
& +\frac{1}{858}(572 \sqrt{2}-156 \sqrt{30}+143 \sqrt{35}+264 \sqrt{42}+3432)(m-1) l \\
\mathrm{GA}_{5}\left(\mathrm{GL}_{m}^{l}\right)= & \frac{1}{5148}(1287 \sqrt{15}+27456 \sqrt{2}+4576 \sqrt{5}+936 \sqrt{30}+8580 \sqrt{35}+13464 \sqrt{42}+205920) m
\end{aligned}
$$

The Sanskruti index SI $\left(\mathrm{GL}_{m}^{l}\right)$ can be calculated by using equation (12) as follows: 


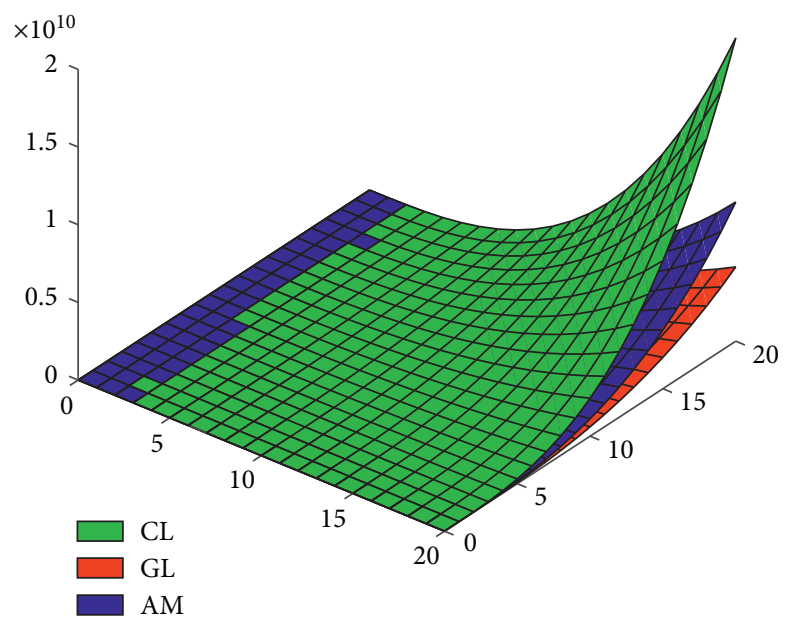

Figure 7: 3D graph of the $M$-polynomial of $\mathrm{CL}_{5}^{15}, \mathrm{GL}_{6}^{3}$, and $\mathrm{AM}_{3}^{5}$.
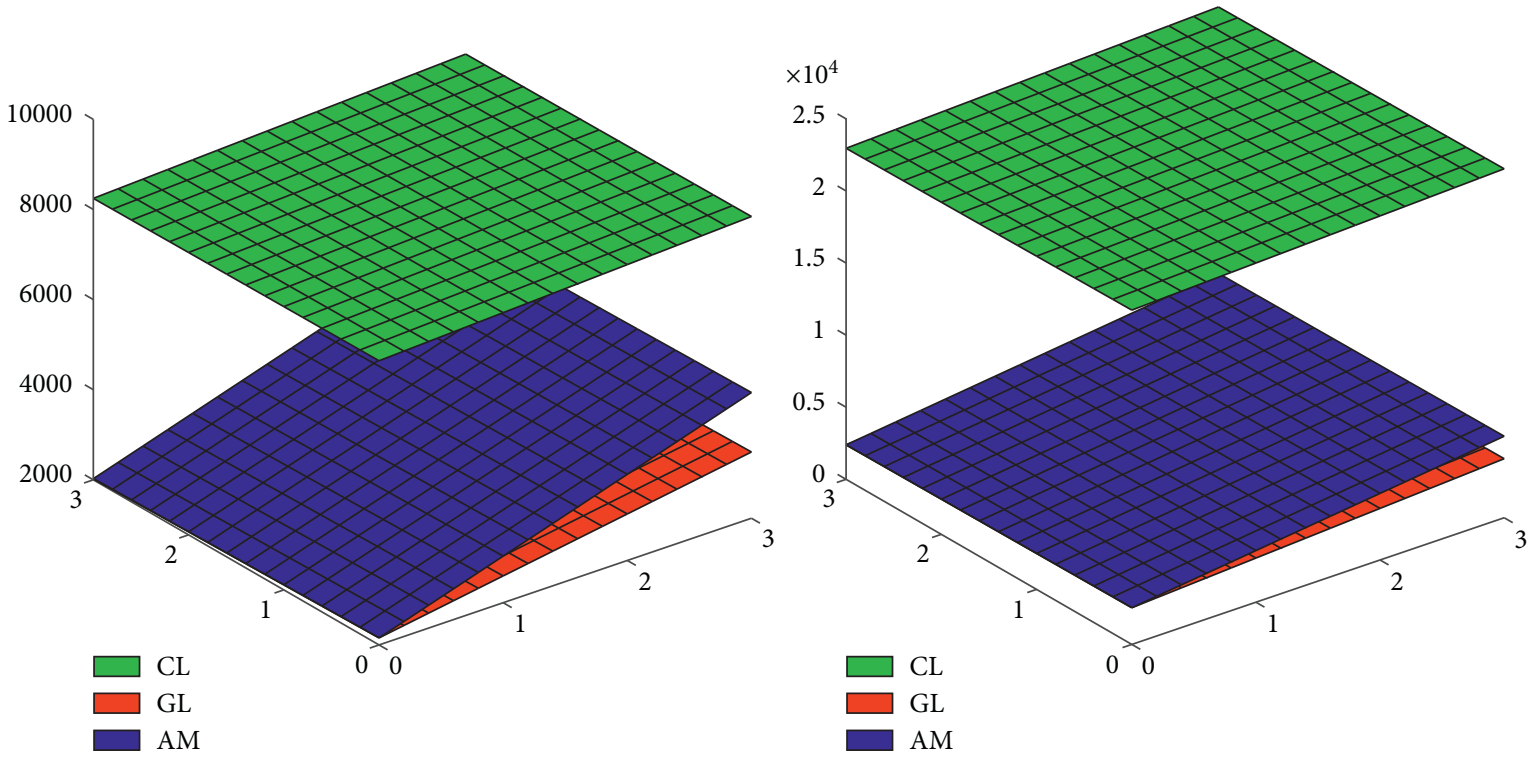

Figure 8: Comparison of $\mathrm{CL}_{m}^{l}, \mathrm{GL}_{m}^{l}$, and $\mathrm{AM}_{m}^{l}$ using $M_{1}$ and $M_{2}$. (a) 3D graphs of the first Zagreb index. (b) 3D graphs of the second Zagreb index.

$$
\text { (3) } \begin{aligned}
\mathrm{SI}\left(\mathrm{GL}_{m}^{l}\right)= & \sum_{v w \in E\left(\mathrm{GL}_{m}^{l}\right)}\left(\frac{s_{v} s_{w}}{s_{v}+s_{w}-2}\right)^{3}=\left|E_{35}\right|\left(\frac{15}{6}\right)^{3} \\
& +\left|E_{36}\right|\left(\frac{18}{7}\right)^{3}+\left|E_{45}\right|\left(\frac{20}{7}\right)^{3}+\left|E_{55}\right|\left(\frac{25}{8}\right)^{3}+\left|E_{56}\right|\left(\frac{30}{9}\right)^{3} \\
& +\left|E_{57}\right|\left(\frac{35}{10}\right)^{3}+\left|E_{66}\right|\left(\frac{36}{10}\right)^{3}+\left|E_{67}\right|\left(\frac{42}{11}\right)^{3}+\left|E_{77}\right|\left(\frac{49}{12}\right)^{3} \\
= & \left(\frac{125}{8}\right)(m+1)+\left(\frac{5832}{343}\right)(8 m+m l-l-1)+\left(\frac{8000}{343}\right)(2 m+2)
\end{aligned}
$$



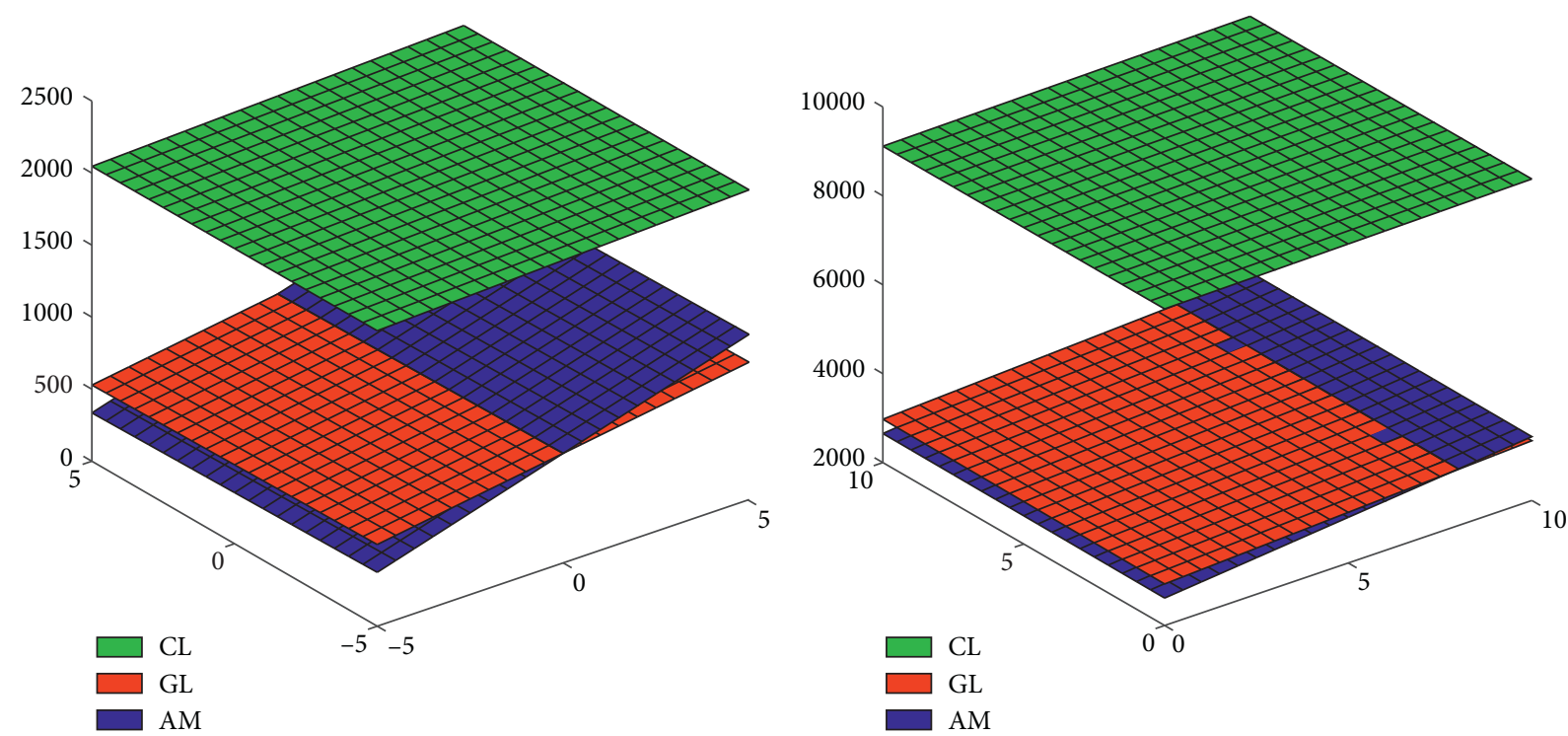

Figure 9: Comparison of $\mathrm{CL}_{m}^{l}, \mathrm{GL}_{m}^{l}$, and $\mathrm{AM}_{m}^{l}$ using $R_{(-1 / 2)}$ and $\chi_{(-1 / 2)}$. (a) 3D graphs of the Randić index. (b) 3D graphs of the sumconnectivity index.
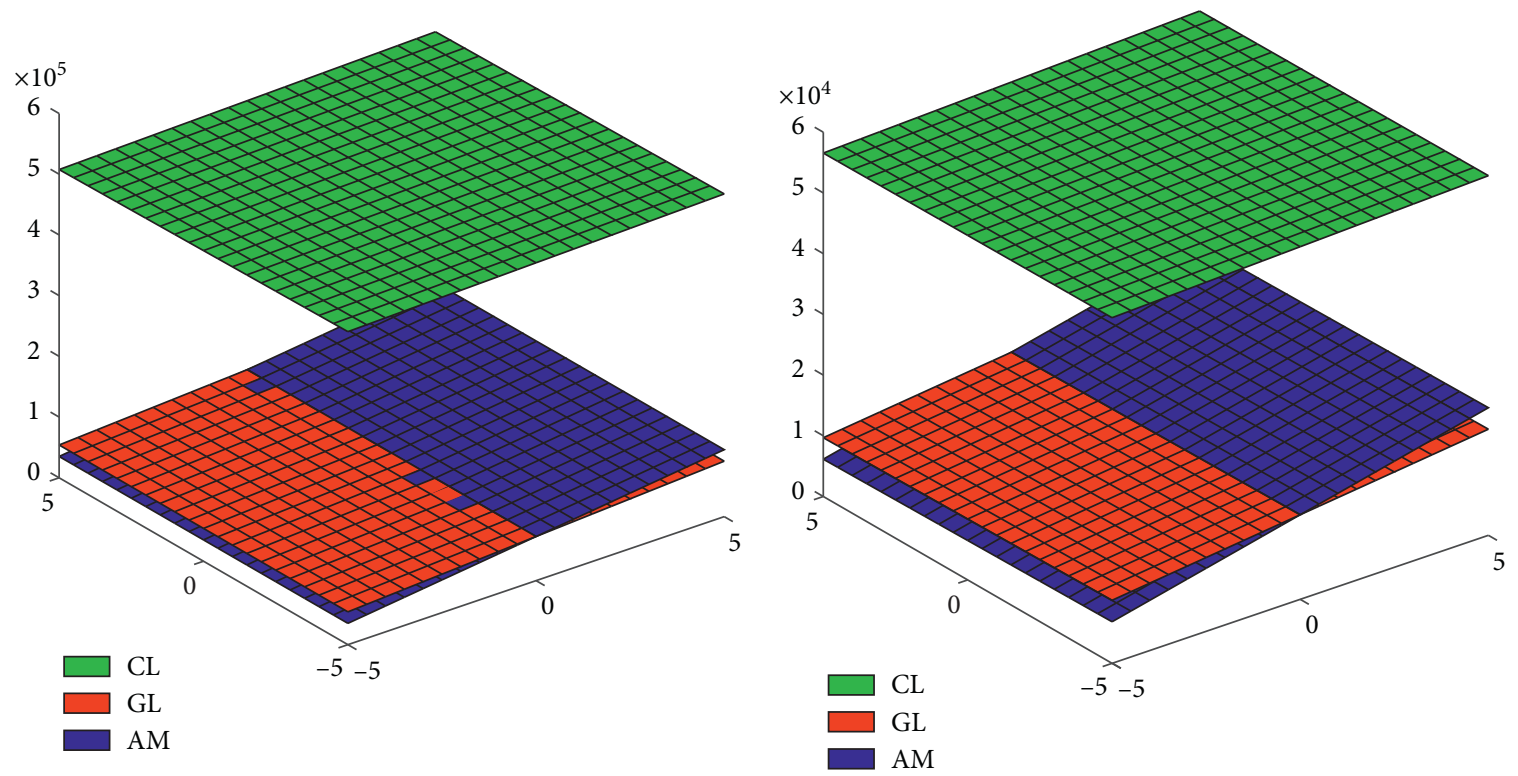

Figure 10: Comparison of $\mathrm{CL}_{m}^{l}, \mathrm{GL}_{m}^{l}$, and $\mathrm{AM}_{m}^{l}$ using Sanskruti and augmented Zagreb indices. (a) 3D graphs of the first SI. (b) 3D graphs of the AZI.

$$
\begin{aligned}
& +\left(\frac{15625}{512}\right)(11 m+m l-l-1)+\left(\frac{1000}{27}\right)(12 m+m l-l)+ \\
& +\left(\frac{343}{8}\right)(10 m+m l-l-2)+\left(\frac{5832}{125}\right)(28 m+3 m l-3 l-4) \\
& +\left(\frac{74088}{1331}\right)(17 m+2 m l-2 l+1)+\left(\frac{117649}{1728}\right)(m-1) \\
& =\left(\frac{2940909850294457}{788889024000}\right) m+\left(\frac{298773851517107}{788889024000}\right)(m-1) l-\left(\frac{216711945260101}{788889024000}\right) .
\end{aligned}
$$



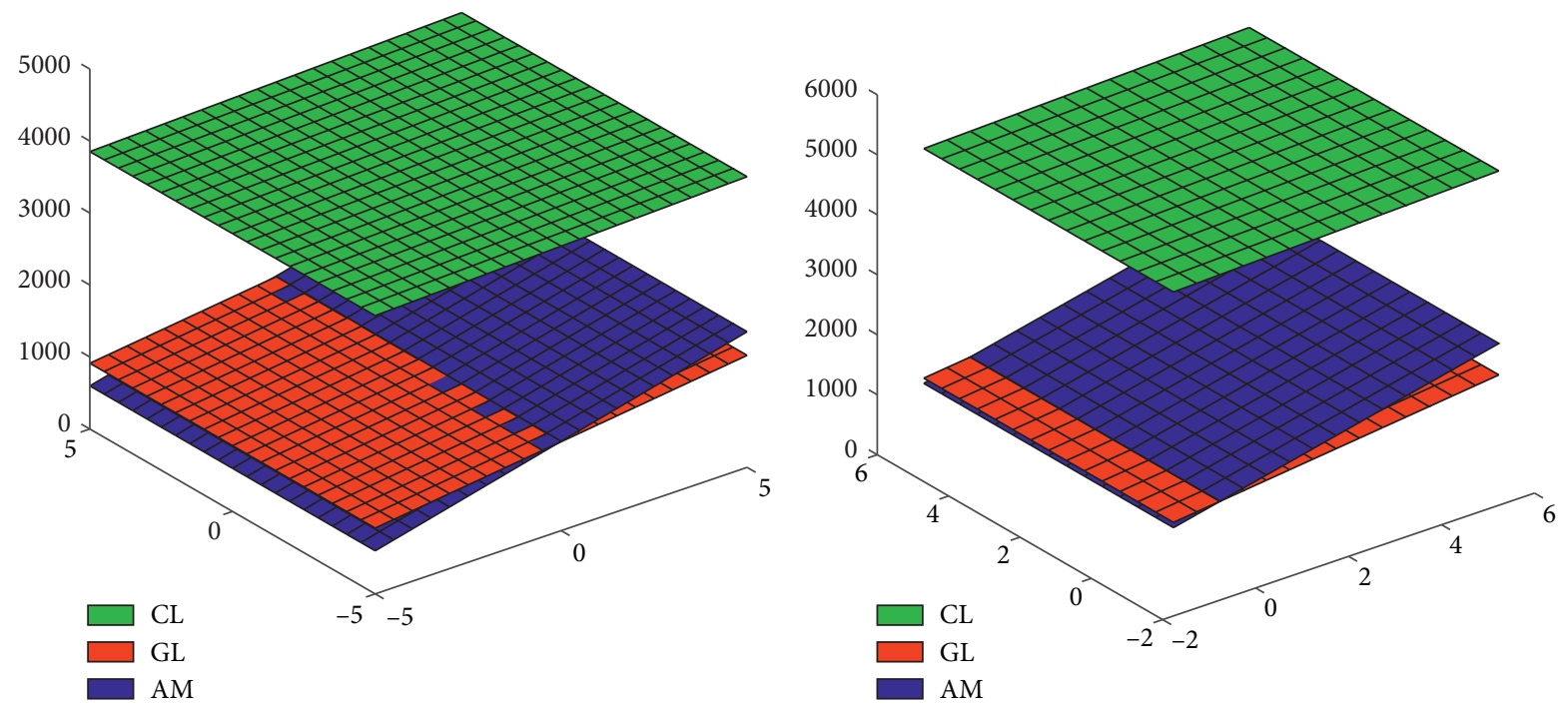

Figure 11: Comparison of $\mathrm{CL}_{m}^{l}, \mathrm{GL}_{m}^{l}$, and $\mathrm{AM}_{m}^{l}$ using $\mathrm{ABC}$ and GA. (a) 3D graphs of the ABC index. (b) 3D graphs of the GA index.
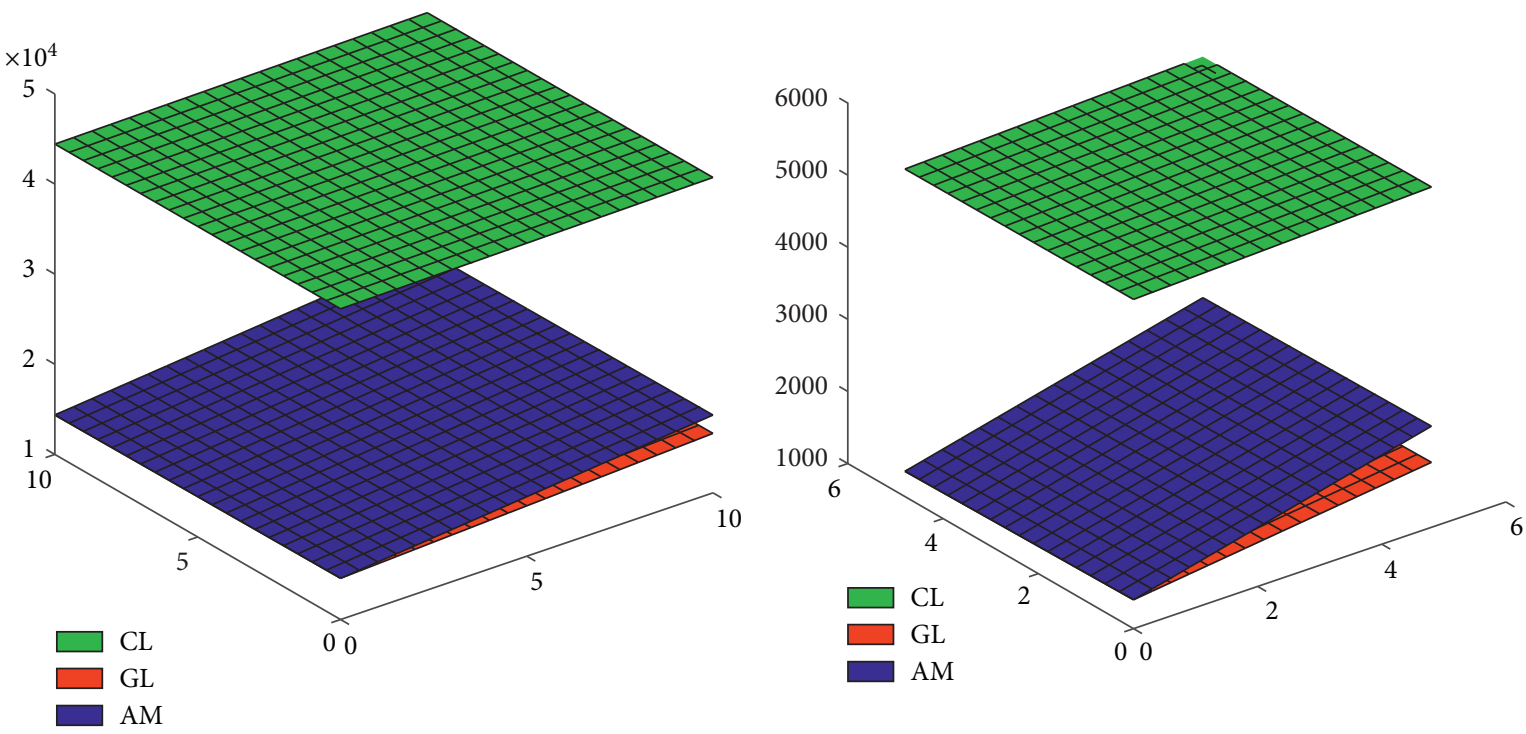

FIGURE 12: Comparison of $\mathrm{CL}_{m}^{l}$, $\mathrm{GL}_{m}^{l}$, and $\mathrm{AM}_{m}^{l}$ using SDD and ISI. (a) 3D graphs of the SDD index. (b) 3D graphs of the ISI.
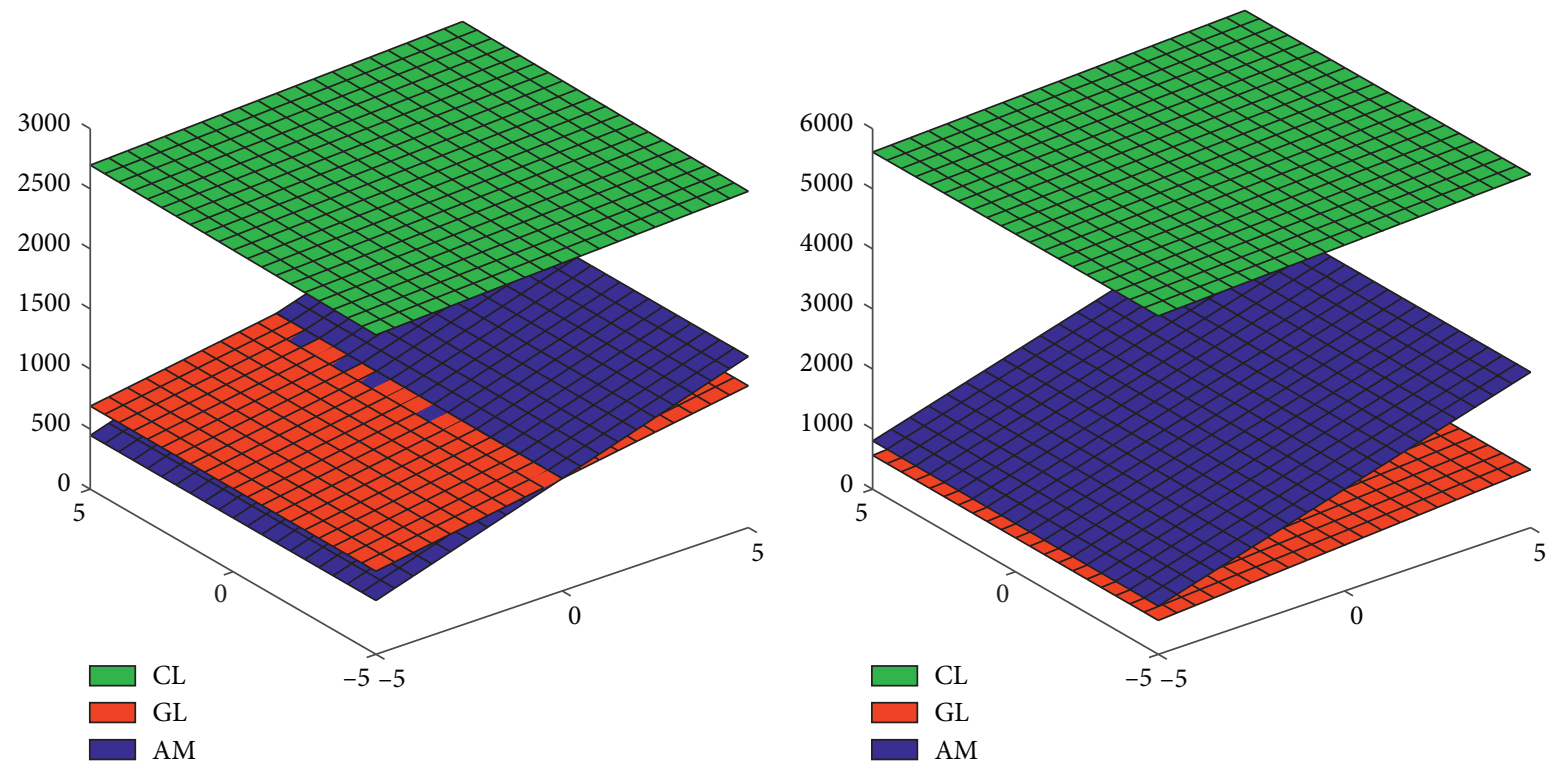

Figure 13: Comparison of $\mathrm{CL}_{m}^{l}, \mathrm{GL}_{m}^{l}$, and $\mathrm{AM}_{m}^{l}$ using $\mathrm{ABC}_{4}$ and $\mathrm{GA}_{5}$. (a) $3 \mathrm{D}$ graphs of the $\mathrm{ABC}_{4}$ index. (b) $3 \mathrm{D}$ graphs of the GA $\mathrm{index}$ 
Theorem 12. Let $A M_{m}^{l}$ be the amylopectin network consisting of $(m-1)$ branches where each branch has length $l$; then, $A B C_{4}, G A_{5}$, and SI of $A M_{m}^{l}$ are given as follows:

$$
\begin{aligned}
\mathrm{ABC}_{4}\left(\mathrm{AM}_{m}^{l}\right)= & \frac{1}{210}(2072 \sqrt{10}+1230 \sqrt{14}+42 \sqrt{35}+1764 \sqrt{2}+462 \sqrt{30}+185 \sqrt{462}+60 \sqrt{3}) m \\
& +\frac{1}{210}(65 \sqrt{14}+84 \sqrt{2}+105 \sqrt{10}+10 \sqrt{462}+21 \sqrt{30})(m-1) l \\
& +\frac{1}{210}(42 \sqrt{35}-98 \sqrt{10}-95 \sqrt{14}-84 \sqrt{2}+5 \sqrt{462}-60 \sqrt{3}) .
\end{aligned}
$$

(2)

$$
\begin{aligned}
\mathrm{GA}_{5}\left(\mathrm{AM}_{m}^{l}\right)= & \frac{1}{468}(117 \sqrt{15}+5616 \sqrt{2}+416 \sqrt{5}+1872 \sqrt{30}+1560 \sqrt{35}+2664 \sqrt{42}+37440) m \\
& +\frac{1}{858}(572 \sqrt{2}+143 \sqrt{35}+156 \sqrt{30}+264 \sqrt{42}+3432)(m-1) l \\
& +\frac{1}{468}(117 \sqrt{15}-312 \sqrt{2}+416 \sqrt{5}+156 \sqrt{35}+72 \sqrt{42}-12168)
\end{aligned}
$$

(3)

$$
\begin{aligned}
\operatorname{SI}\left(\mathrm{AM}_{m}^{l}\right)= & \left(\frac{5928648365465527}{788889024000}\right) m \\
& +\left(\frac{298773851517107}{788889024000}\right)(m-1) l \\
& -\left(\frac{213033772785851}{788889024000}\right) .
\end{aligned}
$$

\section{Concluding Remarks: A Comparative Analysis of Cellulose, Glycogen, and Amylopectin Networks}

Figure 7 demonstrates a comparison between 3D graphs of the $M$-polynomial of cellulose, glycogen, and amylopectin networks (all having the same number of monomers, i.e., 75 hexagons).

Figures 8-13 provide a visual comparison among various TIs of cellulose, glycogen, and amylopectin networks.

Although both natural and synthetic polymers are appropriate for the drug delivery and, in general, for the pharmaceutical industry, however, natural polymers are more suitable as they are nontoxic, biocompatible, without side effects, and economical. As pointed out earlier, $M_{1}, M_{2}$, $R_{(-1 / 2)}$, and Randić-type indices $\left(\chi_{(-1 / 2)}, \mathrm{ABC}\right.$, and $\left.\mathrm{AZI}\right)$ assess the intensity of branching and connectivity in the molecular graph. Figures 8-11 pronounce the subsequent order between the indices for the same number of monomers (i.e., same values of $m$ and $l$ in each polymer). Hence,
$\mathrm{TI}\left(\mathrm{GL}_{m}^{l}\right) \leq \mathrm{TI}\left(\mathrm{AM}_{m}^{l}\right) \leq \mathrm{TI}\left(\mathrm{CL}_{m}^{l}\right) \quad$ where $\quad \mathrm{TI} \in\left\{M_{1}, M_{2}\right.$, $\left.R_{(-1 / 2), \chi_{(-1 / 2)}, \mathrm{ABC}, \mathrm{AZI}}\right\}$. This ordering is convincing due to the fact that there exists extensive cross-linking in cellulose, while glycogen (frequent branching) and amylopectin (less branching) are branched polymers. In [53], properties of the SDD index and ISI are investigated, and it turned out that the ISI and SDD index are reasonable predictors of total surface area for octane isomers and polychlorobiphenyls, respectively. We conjecture, relying on the comparison presented in Figure 12, the relationship between surface areas (SAs) of cellulose, glycogen, and amylopectin which could have been organised as $\mathrm{SA}\left(\mathrm{AM}_{m}^{l}\right) \leq \mathrm{SA}\left(\mathrm{GL}_{m}^{l}\right) \leq$ $\mathrm{SA}\left(\mathrm{CL}_{m}^{l}\right)$, for the same number of monomers.

We observe, from Figures 8-11 and Figure 13, that all the graphs of TIs for cellulose behave like an outlier as compared to glycogen and amylopectin. We anticipate that the eccentric behavior of cellulose is due to its nature of being used as a building material (forms the plant cell wall) as well as its physical properties such as the presence of monomer $\beta$-glucose, insoluble, indigestible, and considerable tensile strength. Moreover, all the graphs of TIs for glycogen and amylopectin go alongside, which might be due to the presence of monomer $\alpha$-glucose, solubility, digestibility, and their nature of being used as energy storage (bonds break easily) in animal organs and plants, respectively. Also, the results obtained in this section could further be applicable in QSPR/QSAR analysis to predict the biological properties of the natural polymers under discussion.

For future research, the study of polysaccharides can be further enhanced to molecular graphs of other natural polymers. Develop molecular graphs for some new natural 
polymers such as proteins and nucleotides (RNA and DNA), and give a mathematical formulation of degree-based indices studied in this article. Finally, compare their physicochemical properties, theoretically and mathematically, using these indices.

\section{Data Availability}

All the data used to support the findings of this study are included within this article. However, the reader may contact the corresponding author for more details of the data.

\section{Conflicts of Interest}

The authors declare no conflicts of interest.

\section{References}

[1] S. C. Basak, G. J. Niemi, and G. D. Veith, "Predicting properties of molecules using graph invariants," Journal of Mathematical Chemistry, vol. 7, no. 1, pp. 243-272, 1991.

[2] S. C. Basak and G. D. Grunwald, "Molecular similarity and estimation of molecular Properties," Journal of Chemical Information and Computer Sciences, vol. 35, no. 3, pp. 366372, 1995.

[3] M. V. Diudea, QSPR/QSAR Studies by Molecular Descriptors, NOVA, New York, NY, USA, 2001.

[4] R. Todeschini and V. Consonni, Handbook of Molecular Descriptors for Chemoinformatics, Wiley VCH, Weinheim, Germany, 2009.

[5] I. Gutman and N. Trinajstić, "Graph theory and molecular orbitals. Total $\varphi$-electron energy of alternant hydrocarbons $\pi$-electron energy of alternant hydrocarbons," Chemical Physics Letters, vol. 17, no. 4, pp. 535-538, 1972.

[6] M. Randic, "Characterization of molecular branching," Journal of the American Chemical Society, vol. 97, no. 23, pp. 6609-6615, 1975.

[7] M. Cavers, S. Fallat, and S. Kirkland, "On the normalized Laplacian energy and general Randić index $R_{-1}$ of graphs," Linear Algebra and Its Applications, vol. 433, no. 1, pp. 172190, 2010.

[8] I. Gutman, B. Furtula, and Ş. B. Bozkurt, "On Randić energy," Linear Algebra and Its Applications, vol. 442, pp. 50-57, 2014.

[9] B. Böllöbás and P. Erdös, "Graphs of extremal weights," Ars Combinatoria, vol. 50, pp. 225-233, 1998.

[10] B. Zhou and N. Trinajstić, "On general sum-connectivity index," Journal of Mathematical Chemistry, vol. 47, no. 1, pp. 210-218, 2010.

[11] G. Shirdel, H. Rezapour, and A. Sayadi, "The hyper-Zagreb index of graph operations," Iranian Journal of Mathematical Chemistry, vol. 4, pp. 213-220, 2013.

[12] X. Li and J. Zheng, "A unified approach to the extremal trees for different indices," MATCH Communications in Mathematical and in Computer Chemistry, vol. 54, pp. 195-208, 2005.

[13] M. Azari and A. Iranmanesh, "Generalized zagreb index of graphs," Studia Universitatis Babes Bolyai, vol. 56, pp. 59-70, 2011.

[14] E. Estrada, L. Torres, L. Rodriguez, and I. Gutman, “An atombond connectivity index: modelling the enthalpy of formation of alkanes," Indian Journal of Chemistry, vol. 37, pp. 849-855, 1998.
[15] E. Estrada, "Atom-bond connectivity and the energetic of branched alkanes," Chemical Physics Letters, vol. 463, no. 4-6, pp. 422-425, 2008.

[16] I. Gutman, J. Tošović, S. Radenković, and S. Marković, "On atom bond connectivity index and its chemical applicability," Indian Journal of Chemistry, vol. 51, pp. 690-694, 2012.

[17] D. Vukičević and B. Furtula, "Topological index based on the ratios of geometrical and arithmetical means of end-vertex degrees of edges," Journal of Mathematical Chemistry, vol. 46, pp. 1369-1376, 2009.

[18] X. Li and Y. Shi, "A survey on the Randić index," MATCH Commun. Math. Comput. Chem.vol. 59, pp. 127-156, 2008.

[19] K. C. Das, I. Gutman, and B. Furtula, "Survey on geometricarithmetic indices of graphs," MATCH Communications in Mathematical and in Computer Chemistry, vol. 65, pp. 595644, 2011.

[20] M. Ghorbani and M. Hosseinzadeh, "Computing ABC4 index of nanostar dendrimers," Optoelectronics and Advanced Materials-Rapid Communications, vol. 4, pp. 1419-1422, 2010.

[21] A. Graovac, M. Ghorbani, and M. A. Hosseinzadeh, "Computing fifth geometric-arithmetic index for nanostar dendrimers," Journal of Mathematical NanoScience, vol. 1, pp. 33-42, 2011.

[22] S. M. Hosamani, "Computing Sanskruti index of certain nanostructures," International Journal of Applied Mathematics and Computer Science, vol. 54, no. 1-2, pp. 425-433, 2017.

[23] P. S. Ranjini, V. Lokesha, and A. Usha, "Relation between phenylene and hexagonal squeeze using harmonic index," International Journal of Graph Theory, vol. 1, pp. 116-121, 2013.

[24] E. Deutsch and S. Klavžar, "M-Polynomial and degree-based topological indices," Iranian Journal of Mathematical Chemistry, vol. 6, pp. 93-102, 2015.

[25] K. V. Camarda and C. D. Maranas, "Optimization in polymer design using connectivity indices," Industrial \& Engineering Chemistry Research, vol. 38, no. 5, pp. 1884-1892, 1999.

[26] W. Wang, X. Hou, and W. Ning, "The k-connectivity index of an infinite class of dendrimer nanostars," Digest Journal of Nanomaterials and Biostructures, vol. 6, pp. 1199-1205, 2011.

[27] A. Ali, A. A. Bhatti, and Z. Raza, "Topological study of treelike polyphenylene systems, spiro hexagonal systems and polyphenylene dendrimer nanostars," Quantum Matter, vol. 5, no. 4, pp. 534-538, 2016.

[28] Z. Shao, P. Wu, X. Zhang, D. Dimitrov, and J.-B. Liu, "On the maximum $\mathrm{ABC}$ index of graphs with prescribed size and without pendent vertices," IEEE Access, vol. 6, pp. 2760427616, 2018.

[29] W. Gao, M. Imran, M. K. Siddiqui, M. Naeem, and F. Jamil, "Molecular description of copper (I) oxide and copper (II) oxide," Quimica Nova, vol. 41, pp. 874-879, 2018.

[30] S. M. Kang, M. K. Siddiqui, N. A. Rehman, M. Naeem, and M. H. Muhammad, "Topological properties of 2-dimensional silicon-carbons," IEEE Access, vol. 6, pp. 59362-59373, 2018.

[31] J.-B. Liu, M. Younas, M. Habib, M. Yousaf, and W. Nazeer, "M-Polynomials and Degree-Based Topological Indices of VC5C7[p,q] and HC5C7[p,q] Nanotubes," IEEE Access, vol. 7, pp. 41125-41132, 2019.

[32] W. Gao, Z. Iqbal, M. Ishaq, A. Aslam, and R. Sarfraz, "Topological aspects of dendrimers via distance based descriptors," IEEE Access, vol. 7, pp. 35619-35630, 2019.

[33] Y. Liu, C. R. Munteanu, E. Fernández Blanco, Z. Tan, A. Santos del Riego, and A. Pazos, "Prediction of nucleotide 
binding peptides using star graph topological indices," Molecular Informatics, vol. 34, no. 11-12, pp. 736-741, 2015.

[34] A. Ali and N. Trinajstić, "A novel/old modification of the first Zagreb index," Molecular informatics, vol. 37, pp. 6-7, 2018.

[35] A. Ali, Z. Du, and K. Shehzadi, "Estimating some general molecular descriptors of saturated hydrocarbons," Molecular Informatics, vol. 38, Article ID e1900007, 2019.

[36] Z. Du, A. Ali, and N. Trinajstić, "Alkanes with the first three maximal/minimal modified first Zagreb connection indices," Molecular Informatics, vol. 38, no. 4, Article ID 1800116, 2019.

[37] S. Hayat, S. Wang, and J.-B. Liu, "Valency-based topological descriptors of chemical networks and their applications," Applied Mathematical Modelling, vol. 60, pp. 164-178, 2018.

[38] M. Arockiaraj, S. Klavžar, J. Clement, S. Mushtaq, and K. Balasubramanian, "Edge distance-based topological indices of strength-weighted graphs and their application to coronoid systems, carbon nanocones and $\mathrm{SiO}_{2}$ nanostructures," Molecular Informatics, vol. 38, Article ID 1900039, 2019.

[39] M. Ahmad, M. Saeed, and M. Javaid, "Comparative study of certain synthetic polymers via bond-additive invariants," IEEE Access, vol. 9, pp. 15388-15403, 2021.

[40] B. Borovicanin, "On the extremal Zagreb indices of trees with given number of segments or given number of branching vertices," MATCH Communications in Mathematical and in Computer Chemistry, vol. 74, pp. 57-79, 2015.

[41] M. Eliasi, A. Iranmanesh, and I. Gutman, "Multiplicative versions of first Zagreb index," MATCH Communications in Mathematical and in Computer Chemistry, vol. 68, pp. 217230, 2012.

[42] I. Gutman, B. Furtula, Z. K. Vukičević, and G. Popivoda, "On Zagreb indices and coindices," MATCH Communications in Mathematical and in Computer Chemistry, vol. 74, pp. 5-16, 2015.

[43] Y. Shi, "Note on two generalizations of the Randić index," Applied Mathematics and Computation, vol. 265, pp. 10191025, 2015.

[44] T. R. Hoare and D. S. Kohane, "Hydrogels in drug delivery: progress and challenges," Polymer, vol. 49, no. 8, pp. 1993-2007, 2008.

[45] V. D. Prajapati, G. K. Jani, N. G. Moradiya, and N. P. Randeria, "Pharmaceutical applications of various natural gums, mucilages and their modified forms," Carbohydrate Polymers, vol. 92, no. 2, pp. 1685-1699, 2013.

[46] N. Ngwuluka, N. Ochekpe, and O. Aruoma, "Naturapolyceutics: the science of utilizing natural polymers for drug delivery," Polymers, vol. 6, no. 5, pp. 1312-1332, 2014.

[47] D. Klemm, B. Heublein, H.-P. Fink, and A. Bohn, "Cellulose: fascinating biopolymer and sustainable raw material," Angewandte Chemie International Edition, vol. 44, no. 22, pp. 3358-3393, 2005.

[48] R. J. Moon, A. Martini, J. Nairn, J. Simonsen, and J. Youngblood, "Cellulose nanomaterials review: structure, properties and nanocomposites," Chemical Society Reviews, vol. 40, no. 7, pp. 3941-3994, 2011.

[49] S. Imran, M. K. Siddiqui, M. Imran, and M. Hussain, "Computing the upper bounds for the metric dimension of cellulose network," Applied Mathematics E-Notes, vol. 19, pp. 585-605, 2019.

[50] J. Jensen, P. I. Rustad, A. J. Kolnes, and Y.-C. Lai, "The role of skeletal muscle glycogen breakdown for regulation of insulin sensitivity by exercise," Frontiers in Physiology, vol. 2, p. 112, 2011.

[51] P. J. Roach, A. A. Depaoli-Roach, T. D. Hurley, and V. S. Tagliabracci, "Glycogen and its metabolism: some new developments and old themes," Biochemical Journal, vol. 441, no. 3, pp. 763-787, 2012.

[52] M. M. Adeva-Andany, M. González-Lucán, C. DonapetryGarcía, C. Fernández-Fernández, and E. AmeneirosRodríguez, "Glycogen metabolism in humans," BBA Clinical, vol. 5, pp. 85-100, 2016.

[53] D. Vukičević and M. Gašperov, "Bond additive modeling 1. Adriatic indices," Croatica Chemica Acta, vol. 83, no. 3, pp. 243-260, 2010. 